\title{
Self-management interventions for people with intellectual disabilities: A systematic review
}

\author{
Janice Sandjojo ${ }^{\mathrm{a}, \mathrm{b}, \mathrm{c}, *}$, Emma G. Eltringham ${ }^{\mathrm{a}}$, Winifred A. Gebhardt ${ }^{\mathrm{a}, \mathrm{b}}$, \\ Aglaia M.E.E. Zedlitz ${ }^{\mathrm{a}, \mathrm{b}}$, Petri J.C.M. Embregts ${ }^{\mathrm{d}}$, Andrea W.M. Evers ${ }^{\mathrm{a}, \mathrm{b}, \mathrm{e}}$ \\ ${ }^{a}$ Leiden University, Institute of Psychology, Health, Medical and Neuropsychology Unit, Leiden, the Netherlands \\ ${ }^{\mathrm{b}}$ Leiden University, Leiden Institute for Brain and Cognition (LIBC), Leiden, the Netherlands \\ ${ }^{\mathrm{c}}$ Raamwerk, Noordwijkerhout, the Netherlands \\ d Tranzo, Tilburg School of Social and Behavioural Sciences, Tilburg University, Tilburg, the Netherlands \\ ${ }^{\mathrm{e}}$ Leiden University Medical Center, Department of Psychiatry, Leiden, the Netherlands
}

\section{A R T I C L E IN F O}

\section{Article history:}

Received 11 December 2018

Received in revised form 4 May 2020

Accepted 6 June 2020

\section{Keywords:}

Behavioural change technique

Intellectual disabilities

Intervention

Review

Self-Management

\begin{abstract}
A B S T R A C T
Objective: People with intellectual disabilities (ID) often experience difficulties managing their affairs. This study reviewed self-management interventions for people with mild to moderate ID, studying interventions' effectiveness and applied behavioural change techniques (BCTs).

Methods: A systematic literature search was conducted in PubMed, PsychINFO, Web of Science, Embase, Emcare, Cochrane, and ProQuest. Data were extracted on study, intervention, and participant characteristics, and results.

Results: Of the 681 studies retrieved, 36 met the inclusion criteria. Most studies used case study designs and small samples. There were eight randomised controlled trials and one non-randomised study. Studies were mostly of moderate quality (Mixed Methods Appraisal Tool). Twenty-two interventions targeted a singular practical skill for a specific context. In allinterventions, the provider applied several BCTs; in 13 studies participants were also trained to apply BCTs themselves. In all studies, improvements in self-management were reported, which mostly maintained over time $(n=20)$. If measured, generalisation to other settings was also found.

Conclusions: Future studies should aim for a higher methodological quality and couldconsider targeting more generic self-management and a wider application of BCTs by people with ID themselves.

Practice implications: The findings suggest that training can promote self-management in people with ID.
\end{abstract}

(C) 2020 Elsevier B.V. All rights reserved.

\section{Contents}

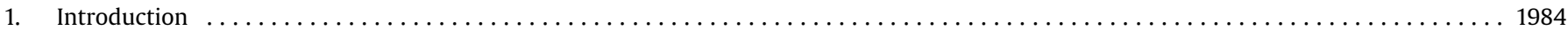

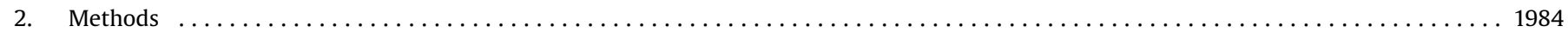

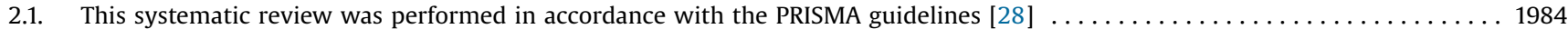

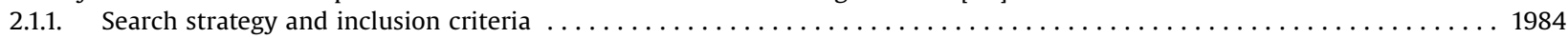

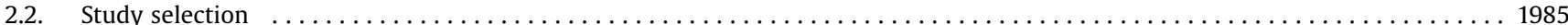

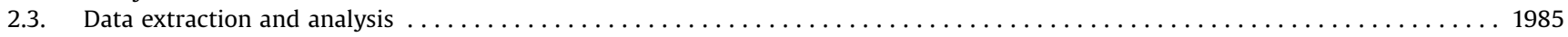

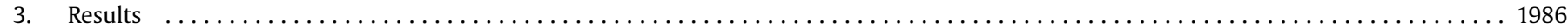

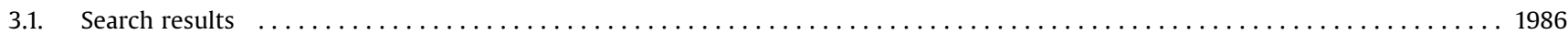

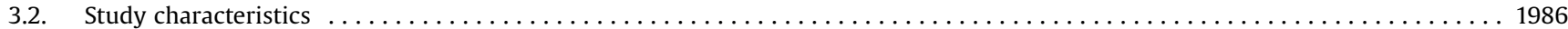

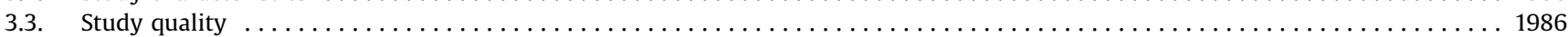

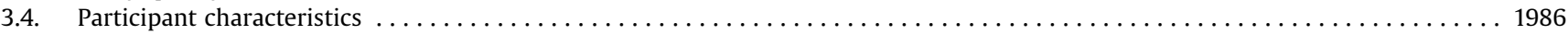

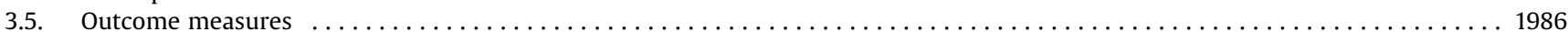

\footnotetext{
* Corresponding author at: Janice Sandjojo, Leiden University, Faculty of Social and Behavioural Sciences, Institute of Psychology, Health, Medical and Neuropsychology Unit, PO Box 9555, 2300 RB, Leiden, the Netherlands.

E-mail address: j.sandjojo@fsw.leidenuniv.nl (J. Sandjojo).
} 


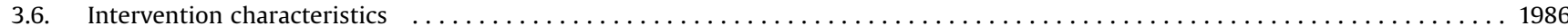

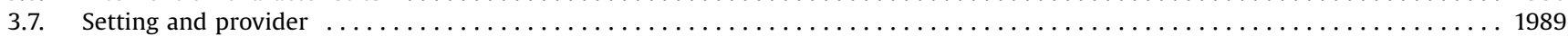

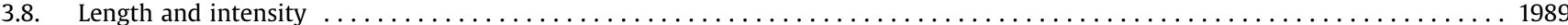

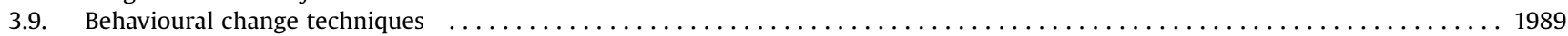

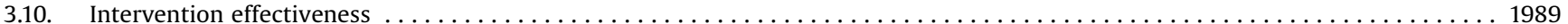

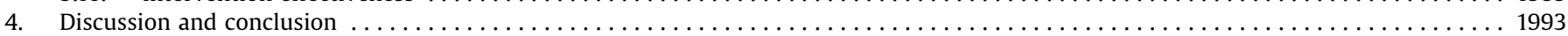

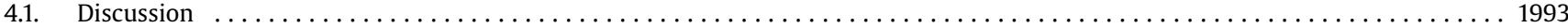

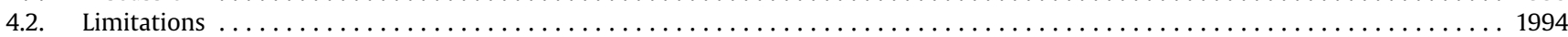

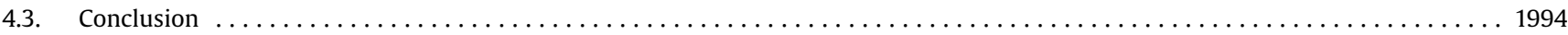

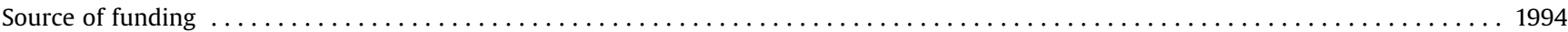

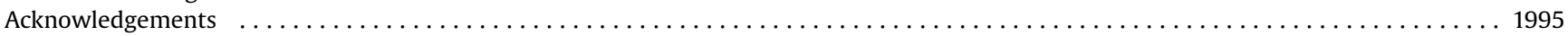

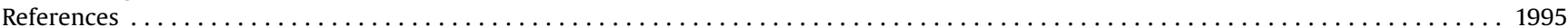

\section{Introduction}

Awareness is increasing that people with intellectual disabilities (ID) should have equal rights and be included as equal cocitizens in society. This is supported by the United Nations [1], which further declare that people with ID should be enabled to live as independently as possible and to be autonomous with respect to making their own decisions. In the Netherlands, this increasing awareness coincides with the emergence of a 'participation society', where citizens, including people with ID, first have to try to arrange their affairs themselves, before they can turn towards the government. However, people with ID commonly have difficulties with self-managing their affairs [2-4], which can vary from difficulties with personal care and household activities, to trouble with recreational activities, community participation, and employment [5-8]. Various studies have shown nonetheless that most people with ID have a desire to manage their activities more independently [2,9-11]. Increasing the abilities of people with ID to handle their affairs themselves could enhance their quality of life and community participation [3,12] and could reduce behavioural problems [13]. Interventions that promote selfmanagement of people with ID are therefore of importance.

Self-management is a broad term that refers to processes and activities that are related to deliberately influencing one's behaviour in order to reach personally desired outcomes [14]. This umbrella term includes being independent in handling one's affairs and in taking care of oneself, thereby solely relying on one's own abilities, efforts, resources, and judgement [15]. Selfmanagement is also strongly related to self-determination, which involves having personal control over making choices and decisions to lead one's life according to one's own preferences, without being completely subjected to external influences [16,17].

Various studies on self-management interventions for people with ID have been conducted and several literature reviews have already collectively analysed some of these previous studies. However, like the individual studies themselves, these reviews only focused on a specific self-management domain such as selfmanagement at work [18-21], self-management of chronic disease $[22,23]$, or the use of activity schedules [24] and self-instructions [8]. Although it was generally found that the self-management interventions reviewed were effective, it is difficult to determine which factors play a key role in the interventions' effectiveness. This is largely due to studies' widely varying self-management goals, outcome measures, and research methods, which hamper thorough comparison. A greater understanding of the key elements of effective self-management interventions could benefit the further development of such interventions and consequently the quality of life of people with ID. In this regard, further identification of the behavioural change techniques (BCTs) applied and their respective effectiveness could contribute to our understanding of how self-management interventions work and how their effects can be optimised [25]. BCTs are active components of an intervention that are designed to alter or redirect causal processes that regulate behaviour [26], such as self-instructions and reinforcements. People with ID can learn to apply BCTs themselves to attain a greater self-management, but they can also be applied by an intervention provider. Recently, Willems, Hilgenkamp, Havik, Waninge and Melville [27] examined how BCTs were applied in interventions for people with ID that targeted physical activity and nutrition. They found that in most cases, several BCTs were applied in the interventions reviewed, such as 'providing information on consequences of behaviour in general' and 'planning social support/social change'. The application of BCTs in self-management interventions for people with ID has not yet been studied.

The aim of the current systematic literature review is to summarise studies that have evaluated the effectiveness of selfmanagement interventions for people with mild to moderate ID. In contrast to the abovementioned reviews that only focused on a certain type of self-management interventions, this review analyses a broad range of interventions that aim to promote self-management in daily life. We aim to examine the BCTs that were used to promote the targeted self-management behaviour, as well as the effectiveness of the interventions. In this way, we aim to create a broader insight into the effects of such self-management interventions for this population.

\section{Methods}

\subsection{This systematic review was performed in accordance with the PRISMA guidelines [28]}

\subsubsection{Search strategy and inclusion criteria}

In order to identify relevant studies for our review, PubMed ( incl. MEDLINE), PsychINFO, Web of Science, Embase, Emcare, COCHRANE Library (incl. CENTRAL), and ProQuest (Social Services Abstracts and Sociological Abstracts) were systematically searched from inception to 18 September 2017. The search strategy was based on the Population, Intervention, Comparison, and Outcome (PICO) approach. Search terms (including major headings, Medical Subject Heading terms, title words, and text words) were used that are indicative of intervention studies (Intervention) aimed at promoting self-management (Outcome) for adults with ID (Population), excluding studies that solely included children or adolescents (see Appendix A).

Studies were included if they evaluated the effect of an intervention for adults with mild to moderate ID that aimed to improve their self-management in daily life. Inclusion criteria concerned that documents were original, peer-reviewed, and published in English (i.e., no reviews, dissertations, and book chapters). Exclusion criteria concerned intervention studies aimed at family, staff, or minors with ID ( $<18$ years). In some studies, not only adults with mild to moderate ID participated, but also minors, adults with severe ID, or people with other disabilities or psychiatric diagnoses. These studies were only included if the 
effects of the intervention on adults with mild to moderate ID could be distinguished from the people in the other groups. Studies were excluded if the interventions were aimed at managing challenging behaviour or emotions, or if outcome measures focused on physical outcomes (e.g., body weight, oral health status). These latter studies were excluded because improvements in physical functioning would not directly indicate improved selfmanagement skills.

\subsection{Study selection}

After excluding all duplicates, retrieved references were loaded into Endnote. Titles and abstracts were independently screened by two reviewers (JS and EE) without blinding to authorship or journal (see Fig. 1). An $83.2 \%$ agreement was achieved. The full texts of the articles that potentially met the criteria were retrieved and examined, including the articles for which there was disagreement. After screening the full texts, reviewers agreed for $95.9 \%$ of the articles that they should be included or excluded. Disagreements between reviewers were discussed until consensus was reached. For three cases for which disagreement remained, two other authors (AZ and WG) were included in the discussion.
The quality of the remaining studies was assessed using the Mixed Methods Appraisal Tool [MMAT; 29]. This was conducted independently by two reviewers (JS and EE), who discussed their judgements afterwards until consensus was reached. Quality criteria could be rated as 'yes' ( 1 point), 'no' ( 0 points), or 'can't tell' (0 points).

\subsection{Data extraction and analysis}

From the studies included, two reviewers (JS and EE) independently extracted information about the study characteristics, participant characteristics, outcome measures, intervention characteristics, BCTs, and main results (both direct and at followup). If information was missing, we tried to retrieve the missing data from the authors. We were able to contact the authors of thirteen articles. One of them replied and provided us with additional information. Regarding the BCTs, for each article we analysed which BCTs were used to target the self-management behaviour and whether these fit the taxonomy of BCTs as described by Michie, Ashford, Sniehotta, Dombrowski, Bishop and French [25]. For 12 articles (33\%), this was done by two reviewers (JS and EE) who initially agreed for $92.1 \%$ of the BCTs and who agreed for

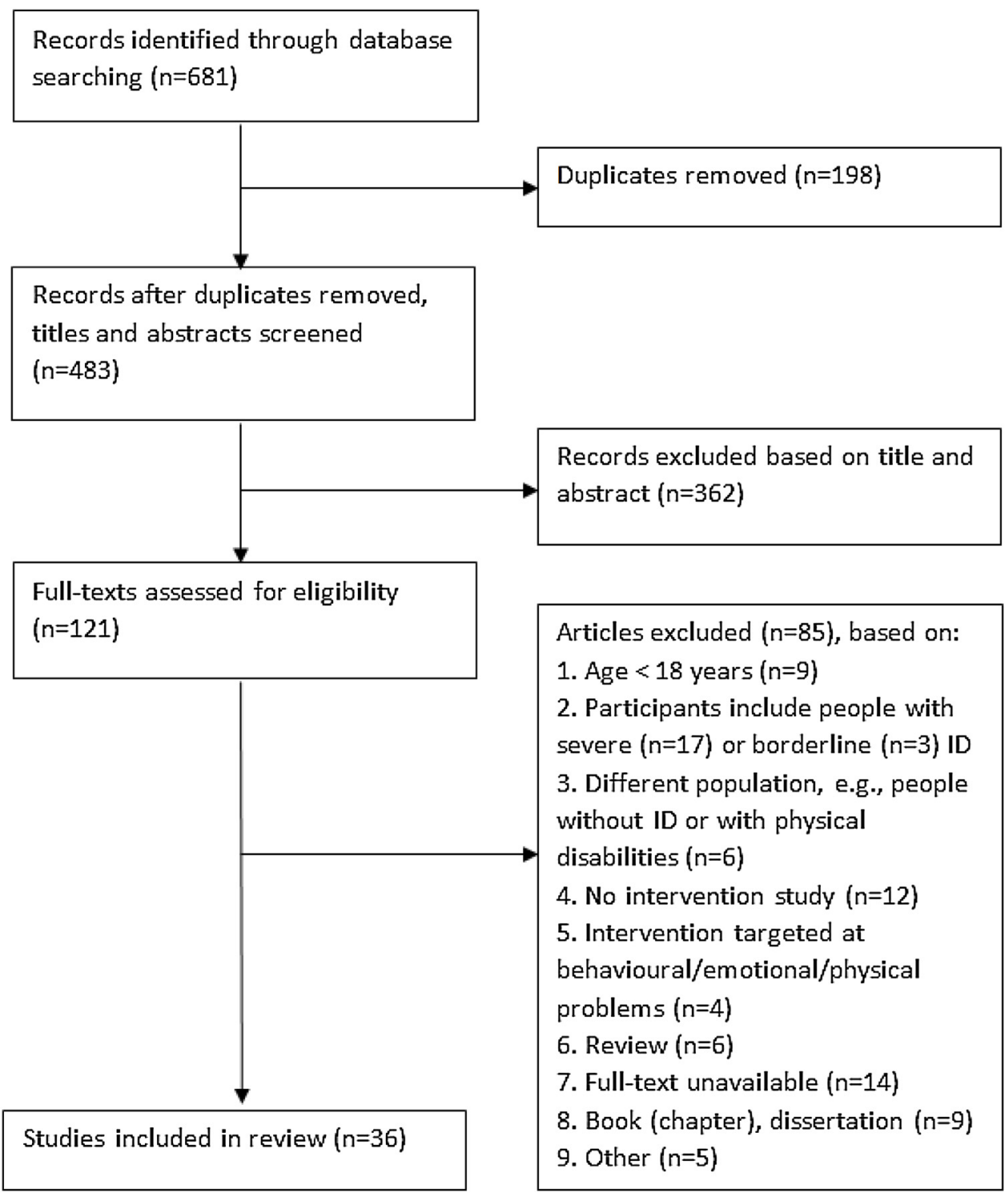

Fig. 1. Flow diagram of the selection process. 
$100 \%$ after discussing the disagreements. Because of the high agreement rate, the other articles were only analysed by one of the two reviewers. Applied BCTs that we came across that were not described in taxonomy of Michie, Ashford, Sniehotta, Dombrowski, Bishop and French [25] were defined separately based on the descriptions in the articles that we reviewed (Appendix B). A distinction was made whether BCTs were applied by the participant (e.g., participants use self-instructions while performing a task) or by the provider of the intervention (e.g., the provider gives verbal instructions on how to perform a task). This allowed us to examine to what extent participants were trained to execute the targeted self-management behaviour completely by themselves or whether they were still dependent on the provider during the intervention.

\section{Results}

After describing the results from our literature search, findings are presented on the study characteristics and the study quality. Next, participant characteristics, outcome measures, intervention characteristics, and findings on effectiveness are described. In spite of our attempts to request missing information from the authors of the studies included, much of these data remained missing.

\subsection{Search results}

The literature search yielded 681 potential publications, of which 483 were unique articles. Of the 121 full texts that were retrieved after screening of the title and abstracts, 36 met our inclusion criteria. Articles were excluded based on a hierarchical approach; if an article was already excluded based on a previous reason, it was not further assessed whether it would also be excluded based on other reasons. Detailed information about the selection process is presented in Fig. 1. Appendix $C$ provides a list of studies that were excluded in the final stage.

\subsection{Study characteristics}

Table 1 presents the characteristics of the 36 articles included. Except for three studies, all were conducted in English speaking countries, of which the United States was the most common $(n=$ $26)$. Other countries of origin were Ireland $(n=3)$, Canada $(n=2)$, Sweden $(n=2)$, Great Britain $(n=1)$, Hong Kong $(n=1)$, and New Zealand $(n=1)$. The majority of articles were published between 1979 and $1999(n=20)$, a few were published in or after $2010(n=$ 5 ). The total number of participants in all 36 studies was 370 . Sample sizes greatly differed between studies, ranging from 1 to 57 . Nineteen studies $(52.8 \%$ ) had less than five participants. There were no dropouts during the period in which interventions were provided, but five studies (article \#4, 8, 11, 14, 30) reported dropouts at follow-up measurements, ranging from $16.6 \%-50 \%$ of the initial sample size. The majority of studies had a (multiple) case study design $(n=24)$. Few studies used a randomised controlled trial $(n=8)$, had a no-treatment control group $(n=5)$, or contained more than one training condition $(n=7)$. In case of the latter, the difference between the conditions concerned for example that more BCTs were applied in one group (e.g., in addition to selfinstruction, also self-evaluation and self-reinforcement), or that one group received in vivo training (training in the real life community) versus conventional classroom training in the other group. Twenty-four studies $(66.7 \%$ ) used multiple baseline measures and 23 studies conducted multiples probes during the course of the intervention. Follow-up data were available for 23 studies (63.9\%). Period of follow-up was generally a couple of months, however this varied from several days to a few years after training. Data regarding the moments of assessment were often not explicitly reported and moments also greatly varied between studies and even within studies, with sometimes some participants being assessed more often than others, with varying periods of time in between.

\subsection{Study quality}

Table 2 and Appendix D show the results of the quality appraisal using the MMAT [29]. Most studies had more nil scores than positive scores, with five studies meeting only one of the five quality criteria (article \#1,14, 17, 22, 30) and 21 studies meeting two out of five. Four studies had positive ratings on three out of five criteria (article \#6,11,16,27), five studies had positive ratings on four out of five criteria (article \#7, 8, 9, 20, 36), and one study had positive ratings on all quality criteria (article \#2), The reason why many studies had lowered ratings was because the sampling strategy, the target population and the sample were not clearly described, and because no statistical analyses were performed. In randomised controlled studies it was often unclear whether the randomisation was correctly performed, whether the groups were comparable at baseline, and whether outcome assessors were blinded to the intervention.

\subsection{Participant characteristics}

Data regarding age were not always complete. In six studies only the average age without a standard deviation was provided, in four only the range. Based on the data that were available, the average age was found to vary between 18.2-50.3 years. Participants' ages ranged from 18 to 64 years. On average, 54.5 $\%$ of participants were female. Most studies included both people with mild and moderate ID ( $n=16)$, instead of solely people with mild $(n=11)$ or moderate ID $(n=9)$. It was, however, not always clear how the level of ID was determined. Data regarding recruitment and inclusion and exclusion criteria were also often not fully reported $(n=33)$.

\subsection{Outcome measures}

The specific outcome measures differed per study, but in most cases $(n=30 ; 83.3 \%)$ it concerned to what extent the selfmanagement behaviour targeted was performed properly (e.g., number of correctly performed steps). In five studies, previously developed instruments (e.g., questionnaires) were used, but whether these were validated measures was not reported (article $\# 1$, 4, 11, 14, 23). Twenty-seven studies (75.0\%) assessed participants' behaviour in their real-life setting.

\subsection{Intervention characteristics}

The majority of interventions ( $n=22$ ) had a specific focus on a practical skill, such as teaching people with ID a singular daily living skill within a certain context. Only six studies targeted several daily living skills (article \#1, 11, 14, 16, 31, 36). The interventions mostly focused on improving self-management at home ( $n=17$; e.g., food preparation, doing laundry) or in the community ( $n=11$; e.g., traveling by bus, doing groceries). Interventions focusing on self-determination or rights were scarce (article \#4, 6, 8) and targeted a specific domain or context (e.g., dealing with health right violations). Two studies focused on selfmanagement at work (article \#9, 35) and another two studies focused on social interaction (article \#20,25). There was one study that aimed for generalisation of self-management skills across situations (article \#16). Studies did not so much use previously established or manualised interventions, but study authors either developed their own intervention or (partially) used an 
Table 1

Main study and participant characteristics of the studies included.

\begin{tabular}{|c|c|c|c|c|c|c|c|c|c|}
\hline \# & $\begin{array}{l}\text { Authors } \\
\text { (year) }\end{array}$ & Country & $\begin{array}{l}\text { Design (Presence of } \\
\text { control group or } \\
\text { multiple training } \\
\text { groups) }\end{array}$ & Setting & $\begin{array}{l}\text { Participants } \\
\text { - } N \\
\text { - Mean (M) age (SD) } \\
\text { - Gender (\% female) } \\
\text { - Level of ID }\end{array}$ & $\begin{array}{l}\text { Target } \\
\text { behaviour }\end{array}$ & $\begin{array}{l}\text { Time-related aspects } \\
\text { - Number of sessions } \\
\text { - Length of session } \\
\text { - Timespan }\end{array}$ & Provider & $\begin{array}{l}\text { Individual or } \\
\text { group } \\
\text { training } \\
\text { (group size) }\end{array}$ \\
\hline 1 & $\begin{array}{l}\text { Crnic et al. } \\
\text { (1979) [46] }\end{array}$ & USA & Unclear (no) & Group home & $\begin{array}{l}n=17 ; M=23.4(n / a) \\
52.9 \% \text { female; mild ID }\end{array}$ & $\begin{array}{l}\text { Independent } \\
\text { living skills } \\
\text { NOS }^{\mathrm{a}}\end{array}$ & $\begin{array}{l}4-18 \text { months }(\text { mean }= \\
12.1)\end{array}$ & Support staff & Individual \\
\hline 2 & $\begin{array}{l}\text { Davies et al. } \\
(2003)[47]\end{array}$ & USA & Within-subjects (no) & Unknown & $\begin{array}{l}n=9 ; \mathrm{M}=25.8(n / a) \\
44.4 \% \text { female; mild ID }\end{array}$ & $\begin{array}{l}\text { Withdraw cash } \\
\text { from ATM }\end{array}$ & $\begin{array}{l}1 \text { session; } 20-45 \\
\text { minutes }\end{array}$ & Unknown & Individual \\
\hline 3 & $\begin{array}{l}\text { Davies et al. } \\
(2010)[48]\end{array}$ & USA & $\begin{array}{l}\text { Between-subjects } \\
\text { (control group) }\end{array}$ & Unknown & $\begin{array}{l}n=23 ; \mathrm{M}=32.0(10.4) ; \\
60.9 \% \text { female; mild- } \\
\text { moderate ID }\end{array}$ & $\begin{array}{l}\text { Navigate a bus } \\
\text { route }\end{array}$ & $\begin{array}{l}1 \text { session; } 30-60 \\
\text { minutes }\end{array}$ & $\begin{array}{l}\text { Hand-held } \\
\text { computer } \\
\text { Researcher }\end{array}$ & Individual \\
\hline 4 & $\begin{array}{l}\text { Dukes et al. } \\
\text { (2009) [39] }\end{array}$ & IRL & $\begin{array}{l}\text { Multiple case study } \\
\text { (no) }\end{array}$ & Unknown & $\begin{array}{l}n=4 ; \mathrm{M}=22.5(0.6) \\
50.0 \% \text { female; } \\
\text { moderate ID }\end{array}$ & $\begin{array}{l}\text { Sexually } \\
\text { related } \\
\text { decision } \\
\text { making }\end{array}$ & $\begin{array}{l}20 \text { sessions; } 45 \mathrm{~min} \text {; } \\
\text { twice a week for } 10 \\
\text { weeks }\end{array}$ & Unknown & Individual \\
\hline 5 & $\begin{array}{l}\text { Faloon et al. } \\
(2008)[36]\end{array}$ & USA & Case study (no) & $\begin{array}{l}\text { Human } \\
\text { services } \\
\text { agency }\end{array}$ & $\begin{array}{l}n=1 ; \text { age }=19 ; 0.0 \% \\
\text { female; mild ID }\end{array}$ & $\begin{array}{l}\text { Use of overt } \\
\text { and covert self- } \\
\text { rules }\end{array}$ & $\begin{array}{l}3-5 \text { sessions per week; } \\
30-40 \text { minutes }\end{array}$ & Experimenter & Individual \\
\hline 6 & $\begin{array}{l}\text { Faw et al. } \\
(1996)[40]\end{array}$ & USA & $\begin{array}{l}\text { Multiple case study } \\
\text { (no) }\end{array}$ & $\begin{array}{l}\text { Group homes } \\
\text { and } \\
\text { simulation } \\
\text { home }\end{array}$ & $\begin{array}{l}n=4 ; \mathrm{M}=22.8(2.2) \\
25.0 \% \text { female; mild- } \\
\text { moderate ID }\end{array}$ & $\begin{array}{l}\text { Self- } \\
\text { determination } \\
\text { skills in } \\
\text { selecting a } \\
\text { home }\end{array}$ & 4 sessions; $1 \mathrm{~h}$ & Trainer & Individual \\
\hline 7 & $\begin{array}{l}\text { Feldman } \\
\text { et al. (1999) } \\
\text { [49] }\end{array}$ & CAN & $\begin{array}{l}\text { Multiple case study } \\
\text { (no) }\end{array}$ & Home & $\begin{array}{l}n=10 ; \mathrm{M}=28(n / a) \\
100 \% \text { female; mild ID }\end{array}$ & $\begin{array}{l}\text { Child-care } \\
\text { skills }\end{array}$ & 1 session & Manual & Individual \\
\hline 8 & $\begin{array}{l}\text { Feldman } \\
\text { et al. }(2012) \\
{[42]}\end{array}$ & CAN & $\begin{array}{l}\text { Randomised } \\
\text { controlled trial } \\
\text { (control group) }\end{array}$ & Unknown & $\begin{array}{l}n=31 ; \mathrm{M}=49.0(7.6) \\
51.6 \% \text { female; mild- } \\
\text { moderate ID }\end{array}$ & $\begin{array}{l}\text { Recognition } \\
\text { and redressing } \\
\text { health rights } \\
\text { violations }\end{array}$ & $\begin{array}{l}\text { Mean number of sessions } \\
=10.89(\mathrm{SD}=3.88) ; 2 \mathrm{~h} ; \\
\text { twice a week }\end{array}$ & Trainer & Group (3) \\
\hline 9 & $\begin{array}{l}\text { Gilson et al. } \\
\text { (2016) [50] }\end{array}$ & USA & Case study (no) & $\begin{array}{l}\text { Internship } \\
\text { job site }\end{array}$ & $\begin{array}{l}n=1 ; \text { age }=22 ; 0.0 \% \\
\text { female; level of ID } \\
\text { unknown }\end{array}$ & $\begin{array}{l}\text { Social } \\
\text { interactions } \\
\text { and task } \\
\text { engagement at } \\
\text { work }\end{array}$ & 30 sessions; $4 \mathrm{~h}$ & Job coach & Individual \\
\hline 10 & $\begin{array}{l}\text { Goodson } \\
\text { et al. (2007) } \\
{[51]}\end{array}$ & USA & $\begin{array}{l}\text { Multiple case study } \\
\text { (no) }\end{array}$ & $\begin{array}{l}\text { Vocational } \\
\text { training } \\
\text { centre }\end{array}$ & $\begin{array}{l}n=4 ; \mathrm{M}=34.8(1.5) \\
0.0 \% \text { female; moderate } \\
\text { ID }\end{array}$ & Setting a table & $\begin{array}{l}\text { 5-6 sessions for video } \\
\text { prompting, } 9-13 \\
\text { sessions for video- } \\
\text { prompting plus error- } \\
\text { correction }\end{array}$ & $\begin{array}{l}\text { Computer } \\
\text { Trainer }\end{array}$ & Individual \\
\hline 11 & $\begin{array}{l}\text { Hällgren } \\
\text { et al. (2005) } \\
{[52]}\end{array}$ & SWE & $\begin{array}{l}\text { Multiple case study } \\
\text { (no) }\end{array}$ & Unknown & $\begin{array}{l}n=6 ; \mathrm{M}=\mathrm{n} / \mathrm{a}(n / a) \\
66.7 \% \text { female; mild- } \\
\text { moderate ID }\end{array}$ & $\begin{array}{l}\text { Activities of } \\
\text { Daily Living } \\
\text { (ADL) }\end{array}$ & 5 sessions; 3 months & $\begin{array}{l}\text { Occupational } \\
\text { therapist }\end{array}$ & Individual \\
\hline 12 & $\begin{array}{l}\text { Johnson et al. } \\
\text { (1981) [53] }\end{array}$ & USA & $\begin{array}{l}\text { Multiple case study } \\
\text { (no) }\end{array}$ & $\begin{array}{l}\text { Sheltered } \\
\text { workshop }\end{array}$ & $\begin{array}{l}n=4 ; \mathrm{M}=32.4(13.7) \\
25.0 \% \text { female; mild- } \\
\text { moderate ID }\end{array}$ & $\begin{array}{l}\text { Cooking skills: } \\
\text { broiling, } \\
\text { baking, boiling }\end{array}$ & $\begin{array}{l}2-12 \text { sessions per } \\
\text { subtask }(\text { mean }=4-6) \\
5-40 \text { minutes }(\text { mean }= \\
17)\end{array}$ & Trainer & Individual \\
\hline 13 & $\begin{array}{l}\text { Katz et al. } \\
(1986)[54]\end{array}$ & NZL & $\begin{array}{l}\text { Multiple case study } \\
\text { (no) }\end{array}$ & Group home & $\begin{array}{l}n=9 ; \mathrm{M}=\mathrm{n} / \mathrm{a}(n / a) \\
55.6 \% \text { female; mild- } \\
\text { moderate ID }\end{array}$ & $\begin{array}{l}\text { Fire-safety } \\
\text { skills }\end{array}$ & $20-30$ sessions & Student & Individual \\
\hline 14 & $\begin{array}{l}\text { Kottorp et al. } \\
\text { (2003) [55] }\end{array}$ & SWE & $\begin{array}{l}\text { Multiple case study } \\
\text { (no) }\end{array}$ & $\begin{array}{l}\text { Disability } \\
\text { Services }\end{array}$ & $\begin{array}{l}n=3 ; M=26.7(3.0) \\
100 \% \text { female; moderate } \\
\text { ID }\end{array}$ & $\begin{array}{l}\text { Activities of } \\
\text { Daily Living } \\
\text { (ADL) }\end{array}$ & $6-10$ sessions; 4 months & $\begin{array}{l}\text { Occupational } \\
\text { therapist }\end{array}$ & Individual \\
\hline 15 & $\begin{array}{l}\text { LaCampagne } \\
\text { et al. (1987) } \\
\text { [56] }\end{array}$ & USA & $\begin{array}{l}\text { Multiple case study } \\
\text { (no) }\end{array}$ & $\begin{array}{l}\text { Day } \\
\text { treatment } \\
\text { centre }\end{array}$ & $\begin{array}{l}n=4 ; M=30.0(5.4) \\
75.0 \% \text { female; mild ID }\end{array}$ & Paying bills & 12 sessions; $1 \mathrm{~h} ; 12$ days & Trainer & Group (4) \\
\hline 16 & $\begin{array}{l}\text { Lovett et al. } \\
(1989) \text { [57] }\end{array}$ & USA & $\begin{array}{l}\text { Between-group } \\
\text { (group 1: self- } \\
\text { recording training, } \\
\text { group 2: self- } \\
\text { recording, self- } \\
\text { evaluation, self- } \\
\text { reinforcement } \\
\text { training }\end{array}$ & Home & $\begin{array}{l}n=9 ; \mathrm{M}=27.0(5.2) \\
55.6 \% \text { female; mild- } \\
\text { moderate ID }\end{array}$ & $\begin{array}{l}\text { Activities of } \\
\text { Daily Living } \\
\text { (ADL) }\end{array}$ & Unknown & Trainer & Individual \\
\hline 17 & $\begin{array}{l}\text { Marchetti } \\
\text { et al. (1983) } \\
{[58]}\end{array}$ & USA & $\begin{array}{l}\text { Randomised } \\
\text { controlled trial } \\
\text { (group 1: classroom } \\
\text { training, group 2: } \\
\text { community training) }\end{array}$ & $\begin{array}{l}\text { Classroom or } \\
\text { community }\end{array}$ & $\begin{array}{l}n=18 ; \mathrm{M}=41.0(n / a) \\
\text { unknown \% female; } \\
\text { mild-moderate ID }\end{array}$ & $\begin{array}{l}\text { Pedestrian } \\
\text { skills }\end{array}$ & $\begin{array}{l}40 \text { sessions; } 1.5 \mathrm{~h} \text {; twice a } \\
\text { week for } 20 \text { weeks }\end{array}$ & Support staff & Group (3) \\
\hline 18 & $\begin{array}{l}\text { Martin et al. } \\
\text { (1987) [59] }\end{array}$ & USA & $\begin{array}{l}\text { Multiple case study } \\
\text { with partial- } \\
\text { sequential } \\
\text { withdrawal (no) }\end{array}$ & Home & $\begin{array}{l}n=3 ; \mathrm{M}=31.0(8.9) ; \\
66.7 \% \text { female; mild- } \\
\text { moderate ID }\end{array}$ & $\begin{array}{l}\text { Preparation of } \\
\text { breakfast and } \\
\text { dinner }\end{array}$ & $\begin{array}{l}50 \text { meals; maximum of } \\
3.5 \text { months }\end{array}$ & Support staff & Individual \\
\hline
\end{tabular}


Table 1 (Continued)

\begin{tabular}{|c|c|c|c|c|c|c|c|c|c|}
\hline$\#$ & $\begin{array}{l}\text { Authors } \\
\text { (year) }\end{array}$ & Country & $\begin{array}{l}\text { Design (Presence of } \\
\text { control group or } \\
\text { multiple training } \\
\text { groups) }\end{array}$ & Setting & $\begin{array}{l}\text { Participants } \\
\text { - } N \\
\text { - Mean (M) age (SD) } \\
\text { - Gender (\% female) } \\
\text { - Level of ID }\end{array}$ & $\begin{array}{l}\text { Target } \\
\text { behaviour }\end{array}$ & $\begin{array}{l}\text { Time-related aspects } \\
\text { - Number of sessions } \\
\text { - Length of session } \\
\text { - Timespan }\end{array}$ & Provider & $\begin{array}{l}\text { Individual or } \\
\text { group } \\
\text { training } \\
\text { (group size) }\end{array}$ \\
\hline 19 & $\begin{array}{l}\text { Matson } \\
(1981)[60]\end{array}$ & USA & $\begin{array}{l}\text { Randomised } \\
\text { controlled trial } \\
\text { (control group) }\end{array}$ & $\begin{array}{l}\text { Outpatient } \\
\text { Clinic and } \\
\text { grocery store }\end{array}$ & $\begin{array}{l}n=20 ; \mathrm{M}=34.0(n / a) \\
50.0 \% \text { female; mild ID }\end{array}$ & $\begin{array}{l}\text { Shopping } \\
\text { behaviour }\end{array}$ & $\begin{array}{l}20 \text { sessions; } 1 \text { h; } 20 \\
\text { weekdays }\end{array}$ & Trainer & Group (5) \\
\hline 20 & $\begin{array}{l}\text { Matson } \\
(1982)[61]\end{array}$ & USA & $\begin{array}{l}\text { Randomised } \\
\text { controlled trial } \\
\text { (control group, group } \\
\text { 1: modelling } \\
\text { training, group 2: } \\
\text { independence } \\
\text { training) }\end{array}$ & Unknown & $\begin{array}{l}n=45 ; M=38.4(n / a) \\
55.6 \% \text { female; mild ID }\end{array}$ & $\begin{array}{l}\text { Phone } \\
\text { conversational } \\
\text { skills }\end{array}$ & $\begin{array}{l}1 \mathrm{~h} \text {; three times per week } \\
\text { for } 2 \text { months }\end{array}$ & Trainer & $\begin{array}{l}\text { Group } \\
(7-8)\end{array}$ \\
\hline 21 & $\begin{array}{l}\text { Matson et al. } \\
\text { (1986) [62] }\end{array}$ & USA & $\begin{array}{l}\text { Multiple case study } \\
\text { (no) }\end{array}$ & $\begin{array}{l}\text { Care } \\
\text { institution } \\
\text { and grocery } \\
\text { stores }\end{array}$ & $\begin{array}{l}n=3 ; \mathrm{M}=41.7(10.6) \\
0.0 \% \text { female; mild- } \\
\text { moderate ID }\end{array}$ & $\begin{array}{l}\text { Computational } \\
\text { and shopping } \\
\text { skills }\end{array}$ & $\begin{array}{l}26 \text { sessions; } 1.5 \mathrm{~h} \text {; twice a } \\
\text { week for } 13 \text { weeks }\end{array}$ & Teacher & Group (3) \\
\hline 22 & $\begin{array}{l}\text { McInerney } \\
\text { et al. (1992) } \\
\text { [63] }\end{array}$ & USA & Within-subjects (no) & $\begin{array}{l}\text { Shopping } \\
\text { malls }\end{array}$ & $\begin{array}{l}n=29 ; \mathrm{M}=\mathrm{n} / \mathrm{a}(n / a) ; \\
69.0 \% \text { female; mild- } \\
\text { moderate ID }\end{array}$ & Use of the bus & $\begin{array}{l}60-90 \text { minutes; } 3-5 \\
\text { times per week (mean }= \\
2.86 \text { sessions per week; } \\
S D=1.50 \text { ) for } 6.58 \text { weeks } \\
\text { on average }(S D=4.19 \text { ) }\end{array}$ & $\begin{array}{l}\text { Occupational } \\
\text { therapist }\end{array}$ & $\begin{array}{l}\text { Group } \\
(4-5)\end{array}$ \\
\hline 23 & $\begin{array}{l}\text { Michie et al. } \\
\text { (1998) [64] }\end{array}$ & GBR & $\begin{array}{l}\text { Randomised block } \\
\text { design (control } \\
\text { group, group 1: } \\
\text { classroom training, } \\
\text { group 2: in vivo } \\
\text { training) }\end{array}$ & Unknown & $\begin{array}{l}n=57 ; \mathrm{M}=36.2(12.7) \\
\text { unknown \% female; } \\
\text { mild-moderate ID }\end{array}$ & $\begin{array}{l}\text { Community } \\
\text { living skills }\end{array}$ & $\begin{array}{l} \pm 180 \text { sessions, twice per } \\
\text { week }\end{array}$ & Unknown & $\begin{array}{l}\text { Group } \\
(4-6)\end{array}$ \\
\hline 24 & $\begin{array}{l}\text { Neef et al. } \\
(1990)[65]\end{array}$ & USA & Case study (no) & $\begin{array}{l}\text { Day } \\
\text { habilitation } \\
\text { centre, } \\
\text { (group) } \\
\text { home, and } \\
\text { laundromat }\end{array}$ & $\begin{array}{l}n=1 ; \text { age }=41 ; 0 \% \\
\text { female; mild-moderate } \\
\text { ID }\end{array}$ & $\begin{array}{l}\text { Laundry skills } \\
\text { (washing and } \\
\text { drying) }\end{array}$ & $\begin{array}{l}3-4 \text { times per week; } \\
\pm 3.4 \text { h for drying and } \\
\pm 36.1 \text { for washing }\end{array}$ & Trainer & Individual \\
\hline 25 & $\begin{array}{l}\text { Ores et al. } \\
(1984)[66]\end{array}$ & USA & $\begin{array}{l}\text { Within-subjects } \\
\text { design (no) }\end{array}$ & Unknown & $\begin{array}{l}n=10 ; \mathrm{M}=\mathrm{n} / \mathrm{a}(n / a) \\
50.0 \% \text { female; } \\
\text { moderate ID }\end{array}$ & $\begin{array}{l}\text { Make a } \\
\text { telephone call }\end{array}$ & $\begin{array}{l}1 \text { session; } 48 \mathrm{~s}-5 \mathrm{~min} \text { and } \\
22 \mathrm{~s} \text { demonstration time, } \\
33 \mathrm{~s}-5 \text { min practice time }\end{array}$ & Researcher & Individual \\
\hline 26 & $\begin{array}{l}\text { Rehfeldt et al. } \\
\text { (2003) [67] }\end{array}$ & USA & $\begin{array}{l}\text { Multiple case study } \\
\text { (no) }\end{array}$ & $\begin{array}{l}\text { Day } \\
\text { treatment }\end{array}$ & $\begin{array}{l}n=1 ; \text { age }=22 ; 0.0 \% \\
\text { female; moderate ID }\end{array}$ & $\begin{array}{l}\text { Making a } \\
\text { sandwich }\end{array}$ & 13 sessions & $\begin{array}{l}\text { Computer } \\
\text { Instruction }\end{array}$ & Individual \\
\hline 27 & $\begin{array}{l}\text { Richman } \\
\text { et al. (1984) } \\
{[68]}\end{array}$ & USA & $\begin{array}{l}\text { Multiple case study } \\
\text { (no) }\end{array}$ & Home & $\begin{array}{l}n=1 ; \text { age }=34 ; 100 \% \\
\text { female; mild-moderate } \\
\text { ID }\end{array}$ & $\begin{array}{l}\text { Menstrual care } \\
\text { skills }\end{array}$ & 5-15 minutes & $\begin{array}{l}\text { Researcher } \\
\text { Support staff }\end{array}$ & Individual \\
\hline 28 & $\begin{array}{l}\text { Risley et al. } \\
\text { (1980) [69] }\end{array}$ & USA & $\begin{array}{l}\text { Multiple case study } \\
\text { (no) }\end{array}$ & $\begin{array}{l}\text { Sheltered } \\
\text { workshop }\end{array}$ & $\begin{array}{l}n=3 ; \mathrm{M}=37.7(13.2) \\
33.3 \% \text { female; mild- } \\
\text { moderate ID }\end{array}$ & $\begin{array}{l}\text { Making an } \\
\text { emergency call }\end{array}$ & $\begin{array}{l}\text { Mean number of sessions } \\
=6 ; 8-35 \text { minutes }(\mathrm{M}= \\
13) ; 5 \text { per week }\end{array}$ & Trainer & Individual \\
\hline 29 & $\begin{array}{l}\text { Sarber et al. } \\
\text { (1983) [70] }\end{array}$ & USA & Case study (no) & $\begin{array}{l}\text { Home and } \\
\text { supermarket }\end{array}$ & $\begin{array}{l}n=1 ; \text { age }=34 ; 100 \% \\
\text { female; mild ID }\end{array}$ & $\begin{array}{l}\text { Menu planning } \\
\text { and grocery } \\
\text { shopping }\end{array}$ & Unknown & Counsellor & Individual \\
\hline 30 & $\begin{array}{l}\text { Sigafoos et al. } \\
(2005)[3]\end{array}$ & USA & $\begin{array}{l}\text { Multiple case study } \\
\text { (no) }\end{array}$ & $\begin{array}{l}\text { Vocational } \\
\text { programme }\end{array}$ & $\begin{array}{l}n=3 ; \mathrm{M}=35.3(S D= \\
1.2) ; 0.0 \% \text { female; } \\
\text { moderate ID }\end{array}$ & $\begin{array}{l}\text { Make a bag of } \\
\text { microwave } \\
\text { popcorn }\end{array}$ & $\begin{array}{l} \pm 25 \text { sessions; } 6-8 \\
\text { minutes; twice per week }\end{array}$ & $\begin{array}{l}\text { Computer } \\
\text { Trainer }\end{array}$ & Individual \\
\hline 31 & $\begin{array}{l}\text { Taber- } \\
\text { Doughty }\end{array}$ & USA & $\begin{array}{l}\text { Multiple case study } \\
\text { with alternating }\end{array}$ & Home & $\begin{array}{l}n=4 ; \mathrm{M}=49.3(5.9) \\
25.0 \% \text { female; mild- }\end{array}$ & $\begin{array}{l}\text { Household } \\
\text { tasks }\end{array}$ & 20 sessions; $5-7$ days & $\begin{array}{l}\text { Support staff } \\
\text { Telecare staff }\end{array}$ & Individual \\
\hline
\end{tabular}
treatment [71] (group 1: telecare support, group 2: standard care staff)

32 Tam et al. HKG Quasi-experimental

conventional training, group 2: Virtual Reality training)

33 Taylor et al. IRL Multiple case study (1997) [73] with multi-element phases (no)

34 Taylor et al. IRL Multiple case study (2000) [74]

(group 1: Stimulus Equivalence Training, group 2: Single Instance training, group 3: Multiple Exemplar training)

Vocational $\quad n=16 ; \mathrm{M}=18.2(2.3) ; \quad$ Supermarket skills training $50.0 \%$ female; centre moderate ID shopping

Control group: two 30 min sessions VR group: one $45 \mathrm{~min}$ sessions and one $30 \mathrm{~min}$ session

training

$n=7 ; \mathrm{M}=28.7$ (5.6); $\quad$ Shopping

3-4 sessions per week Trainer

Group

centre and supermarkets

Vocational $\quad n=6 ; \mathrm{M}=27.2$ (5.9); $\quad$ Supermarket

$\begin{array}{lll}\text { Vocational } & n=6 ; \mathrm{M}=27.2(5.9) ; & \text { Supermarket } \\ \text { training } & 66.7 \% \text { female; mild ID } & \text { shopping }\end{array}$

centre and supermarkets
$50.0 \%$ female; mild ID

\pm 32 sessions; $45 \mathrm{~min}$

(individual session) and

90 min (group training);

4 days a week for 2

months
Individual

Group (6) 
Table 1 (Continued)

\begin{tabular}{|c|c|c|c|c|c|c|c|c|c|}
\hline \# & $\begin{array}{l}\text { Authors } \\
\text { (year) }\end{array}$ & Country & $\begin{array}{l}\text { Design (Presence of } \\
\text { control group or } \\
\text { multiple training } \\
\text { groups) }\end{array}$ & Setting & $\begin{array}{l}\text { Participants } \\
\text { - } N \\
\text { - Mean (M) age (SD) } \\
\text { - Gender (\% female) } \\
\text { - Level of ID }\end{array}$ & $\begin{array}{l}\text { Target } \\
\text { behaviour }\end{array}$ & $\begin{array}{l}\text { Time-related aspects } \\
\text { - Number of sessions } \\
\text { - Length of session } \\
\text { - Timespan }\end{array}$ & Provider & $\begin{array}{l}\text { Individual or } \\
\text { group } \\
\text { training } \\
\text { (group size) }\end{array}$ \\
\hline 35 & $\begin{array}{l}\text { Wacker et al. } \\
\text { (1986) [75] }\end{array}$ & USA & Case study (no) & $\begin{array}{l}\text { School and } \\
\text { job site }\end{array}$ & $\begin{array}{l}n=1 ; \text { age }=19) ; 100 \% \\
\text { female; moderate ID }\end{array}$ & Clerical Tasks & 40 sessions; $2 \mathrm{~h}$ & $\begin{array}{l}\text { Job } \\
\text { coordinator }\end{array}$ & Individual \\
\hline 36 & $\begin{array}{l}\text { Wu et al. } \\
(2016)[76]\end{array}$ & USA & $\begin{array}{l}\text { Multiple case study } \\
\text { (no) }\end{array}$ & School & $\begin{array}{l}n=2 ; \mathrm{M}=18.5(0.7) \\
50 \% \text { female; mild- } \\
\text { moderate ID }\end{array}$ & $\begin{array}{l}\text { Daily living } \\
\text { skills }\end{array}$ & $\begin{array}{l}13 \text { or } 40 \text { sessions; } 15-30 \\
\text { minutes }\end{array}$ & Trainer & Individual \\
\hline
\end{tabular}

$\mathrm{n} / \mathrm{a}=$ not available.

${ }^{a}$ Not Otherwise Specified.

intervention that had been previously studied (article \#1, 4, 8, 10, $13,14,18-21,27)$. Interventions widely differed from one another, using for example classroom training, written or pictorial instructions, video demonstrations, simulations, or electronic devices. Interventions were mostly provided on an individual basis ( $n=26$ ), with ten studies using group interventions (range $3-$ 8 participants in a group). Five studies explicitly reported some kind of tailoring within their intervention (e.g., tailoring to individual learning preferences), but it is possible that other interventions were also (partially) tailored, especially those that were provided individually.

\subsection{Setting and provider}

The setting of the interventions varied between studies, with half of the interventions (partially) taking place in the real life setting of the participants (e.g., at home or at work), thereby fostering the transfer of learnt skills to daily life. It widely varied between studies who the provider of the intervention was (e.g., trainer) and most studies $(n=29)$ did not specify how this person was instructed to provide the training and what his or her qualifications were.

\subsection{Length and intensity}

The number and duration of training sessions greatly differed between interventions and data about this were often incomplete. For example, the number of sessions varied from 1 to 180 over a period of 1 day to 18 months and information about this was missing for at least half of the studies. Session length was mostly less than $1(n=9)$ or $2 \mathrm{~h}(n=8)$, although for 16 studies no data were available. Three studies provided 'booster' sessions for participants who were lagging behind (article \#8, 16, 26) and four studies provided follow-up sessions after the training (article \#1, 6, 12, 36).

\subsection{Behavioural change techniques}

To obtain an overview of the BCTs that were applied to attain the targeted self-management behaviour, we analysed per study which antecedent BCTs preceded the desired self-management behaviour of participants and which consequent BCTs followed afterwards [Appendix B; [25] [30],]. We also made a distinction whether BCTs were applied by the participant or by the provider of the intervention (Table 2).

All interventions aimed to promote self-management by means of the provider of the intervention, who applied a range of BCTs to help the participants reach the targeted self-management behaviour $(n=34)$. A common combination of BCTs preceding the desired self-management behaviour of participants (12/36 studies, $33.3 \%$ ) concerned the provider modelling the targeted behaviour or skill, giving instructions, and providing prompts (e.g., a visual/auditory cue, least-to-most prompting). These three BCTs were not only provided verbally, but sometimes also visually (e.g., with the use of a pictorial manual, videos, or gestures). In nine studies, the provider encouraged the generalisation of the targeted selfmanagement behaviour to another situation (e.g., a different supermarket; article \#6, 8, 17, 22, 24, 28, 33, 36). Less frequently antecedent BCTs applied included chaining (article \#15, 22, 23, 27), physical guidance (e.g., holding someone's hand while executing a task; article \#12, 13, 28, 34, 36), and role-play (article \#6, 8, 9, 23, $32,34)$. Consequent BCTs that were applied by the provider that followed the execution of the desired self-management behaviour mostly concerned giving feedback $(n=29)$, which could be further distinguished into praise, corrective feedback, or descriptive feedback. Often a combination of these types of feedback was used (16/36 studies, $44.4 \%)$. In nine cases, some kind of reinforcement was provided (e.g., a consumable or activity; article $\# 7,12,13,16,17,20,21,23,32)$.

Several studies $(n=13)$ trained participants to apply BCTs themselves to attain the targeted self-management behaviour. The antecedent BCTs that were taught concerned the use of selfinstructions (article \# 5, 7, 18, 23, 33, 36) or environmental cues (article \#3, 22). Consequent BCTs applied by participants regarded some form of self-recording or self-monitoring of the performed self-management behaviour, followed by self-evaluation of the performance and self-reinforcement (article \#16,19, 20, 21), or praise (article $\# 5,13,33$ ).

\subsection{Intervention effectiveness}

Although quantitative data on results was missing or incomplete in almost half of the studies, all studies reported that the interventions applied were effective, which generally meant that participants were better able to execute the self-management behaviour targeted properly and independently after training. Twenty-four studies collected follow-up data and twenty of these found that training effects maintained over the follow-up period. All 15 studies ( $41.7 \%$ ) that examined whether participants were able to execute the self-management behaviour trained in other settings (e.g., doing groceries at an unfamiliar supermarket) found evidence for such generalisation effects. Studies that included a notreatment control group all found that participants from the training groups performed better than the control group (article $\# 3,8,19,20,23$ ), both immediately after training as well as at follow-up (in case follow-up data were available). In studies with several training groups, results were mixed. Two studies only reported a significant improvement in the community or in vivo training group, but not in the classroom group (article \# 17, 23). Other studies found that training groups improved equally (article \# 32,34 ) or that both training groups improved performance but 
Table 2

Intervention characteristics of the included studies.

\begin{tabular}{|c|c|c|c|c|c|c|c|c|}
\hline \multirow[t]{3}{*}{$\#$} & \multirow{3}{*}{$\begin{array}{l}\text { Authors } \\
\text { (year) }\end{array}$} & \multirow[t]{3}{*}{ Target behaviour } & \multicolumn{4}{|c|}{ Behaviour Change Techniques } & \multirow[t]{3}{*}{ Main results } & \multirow{3}{*}{$\begin{array}{l}\text { Quality } \\
\text { appraisal }\end{array}$} \\
\hline & & & \multicolumn{2}{|c|}{ Applied by participant } & \multicolumn{2}{|c|}{ Applied by provider } & & \\
\hline & & & Antecedent & Consequent & Antecedent & Consequent & & \\
\hline 1 & $\begin{array}{l}\text { Crnic et al. } \\
(1979)[46]\end{array}$ & $\begin{array}{l}\text { Independent } \\
\text { living skills NOS }\end{array}$ & Unknown & Unknown & Unknown & Unknown & $\begin{array}{l}\text { Outcome: Improved skills in personal } \\
\text { maintenance, clothing care, home } \\
\text { maintenance, food preparation, time } \\
\text { management, social behaviour, } \\
\text { community utilisation, communication, } \\
\text { and academic functioning. } \\
\text { Generalisation to daily life: yes } \\
\text { Follow-up: not measured }\end{array}$ & $1 / 5$ \\
\hline 2 & $\begin{array}{l}\text { Davies et al. } \\
(2003) \text { [47] }\end{array}$ & $\begin{array}{l}\text { Withdraw cash } \\
\text { from ATM }\end{array}$ & $\mathrm{n} / \mathrm{a}$ & $\mathrm{n} / \mathrm{a}$ & $\begin{array}{l}\text { Instruction } \\
\text { Modelling } \\
\text { Prompts }\end{array}$ & Feedback $\left(\right.$ NOS $\left.^{\mathrm{a}}\right)$ & $\begin{array}{l}\text { Outcome: Fewer required help prompts } \\
\text { and fewer errors. } \\
\text { Generalisation to daily life: yes } \\
\text { Follow-up: not measured }\end{array}$ & $5 / 5$ \\
\hline 3 & $\begin{array}{l}\text { Davies et al. } \\
(2010) \text { [48] }\end{array}$ & $\begin{array}{l}\text { Navigate a bus } \\
\text { route }\end{array}$ & $\begin{array}{l}\text { Use of cues } \\
\text { (pictures and } \\
\text { audio } \\
\text { messages) }\end{array}$ & $\mathrm{n} / \mathrm{a}$ & $\begin{array}{l}\text { Instruction } \\
\text { Prompts }\end{array}$ & $\begin{array}{l}\text { Feedback } \\
\text { (descriptive) }\end{array}$ & $\begin{array}{l}\text { Outcome: Experimental group was more } \\
\text { successful at completing a bus route, } \\
\text { made less errors and needed less } \\
\text { landmarking prompts vs. control group. } \\
\text { Generalisation to daily life: yes } \\
\text { Follow-up: not measured }\end{array}$ & $2 / 5$ \\
\hline 4 & $\begin{array}{l}\text { Dukes et al. } \\
\text { (2009) [39] }\end{array}$ & $\begin{array}{l}\text { Sexually related } \\
\text { decision making }\end{array}$ & Unknown & Unknown & Unknown & Unknown & $\begin{array}{l}\text { Outcome: Improved knowledge of human } \\
\text { sexuality and safety practices and } \\
\text { improved decision-making capacity. } \\
\text { Generalisation to daily life: not measured } \\
\text { Follow up: Maintenance of effects for } \\
\text { safety practices, some decay in } \\
\text { knowledge. }\end{array}$ & $2 / 5$ \\
\hline 5 & $\begin{array}{l}\text { Faloon et al. } \\
(2008) \text { [36] }\end{array}$ & $\begin{array}{l}\text { Use of overt and } \\
\text { covert self-rules }\end{array}$ & Self-instruction & $\begin{array}{l}\text { Feedback } \\
\text { (praise) }\end{array}$ & $\begin{array}{l}\text { Instruction } \\
\text { Modelling } \\
\text { Prompts }\end{array}$ & $\begin{array}{l}\text { Feedback } \\
\text { (corrective, } \\
\text { praise) }\end{array}$ & $\begin{array}{l}\text { Outcome: Accuracy improved after overt } \\
\text { and covert self-instruction training. } \\
\text { Performance decreased during overt and } \\
\text { covert blocking sessions. } \\
\text { Generalisation to daily life: yes } \\
\text { Follow-up: not measured }\end{array}$ & $2 / 5$ \\
\hline 6 & $\begin{array}{l}\text { Faw et al. } \\
(1996) \text { [40] }\end{array}$ & $\begin{array}{l}\text { Self- } \\
\text { determination } \\
\text { skills in selecting } \\
\text { a home }\end{array}$ & $\mathrm{n} / \mathrm{a}$ & $\mathrm{n} / \mathrm{a}$ & $\begin{array}{l}\text { Generalisation } \\
\text { Instruction } \\
\text { Modelling } \\
\text { Prompts } \\
\text { Roleplay }\end{array}$ & $\begin{array}{l}\text { Feedback } \\
\text { (descriptive, } \\
\text { instructive, } \\
\text { praise) }\end{array}$ & $\begin{array}{l}\text { Outcome: Increase in skills regarding } \\
\text { asking preference questions, reporting } \\
\text { information, and evaluating homes. } \\
\text { Generalisation to daily life: yes } \\
\text { Follow up: Performances were } \\
\text { maintained. }\end{array}$ & $3 / 5$ \\
\hline 7 & $\begin{array}{l}\text { Feldman } \\
\text { et al. (1999) } \\
{[49]}\end{array}$ & Child-care skills & Self-instruction & $\mathrm{n} / \mathrm{a}$ & Instruction & $\begin{array}{l}\text { Feedback }\left(\mathrm{NOS}^{\mathrm{a}}\right) \text {, } \\
\text { Modelling, } \\
\text { Reinforcement } \\
\left(\mathrm{NOS}^{\mathrm{a}}\right)\end{array}$ & $\begin{array}{l}\text { Outcome: Increased child-care skills to } \\
\text { normal levels for most mothers and child- } \\
\text { care skills. Higher mean percentage } \\
\text { correct after training. } \\
\text { Generalisation to daily life: yes } \\
\text { Follow-up: Even higher mean percentage } \\
\text { correct, skills were maintained. }\end{array}$ & $4 / 5$ \\
\hline 8 & $\begin{array}{l}\text { Feldman } \\
\text { et al. (2012) } \\
{[41]}\end{array}$ & $\begin{array}{l}\text { Recognition and } \\
\text { redressing health } \\
\text { rights violations }\end{array}$ & $\mathrm{n} / \mathrm{a}$ & $\mathrm{n} / \mathrm{a}$ & $\begin{array}{l}\text { Generalisation } \\
\text { Instruction } \\
\text { Modelling } \\
\text { Prompts } \\
\text { Roleplay }\end{array}$ & Feedback (praise) & $\begin{array}{l}\text { Outcome: Training group had more } \\
\text { correct responses to video scenarios } \\
\text { showing health rights, respect, or } \\
\text { responsibility situations vs. control group. } \\
\text { Generalisation to daily life: yes } \\
\text { Follow-up: Improvements were } \\
\text { maintained. }\end{array}$ & $4 / 5$ \\
\hline 9 & $\begin{array}{l}\text { Gilson et al. } \\
\text { (2016) [50] }\end{array}$ & $\begin{array}{l}\text { Social } \\
\text { interactions and } \\
\text { task engagement } \\
\text { at work }\end{array}$ & $\mathrm{n} / \mathrm{a}$ & $\mathrm{n} / \mathrm{a}$ & $\begin{array}{l}\text { Instruction } \\
\text { Modelling } \\
\text { Prompts } \\
\text { Roleplay }\end{array}$ & $\begin{array}{l}\text { Feedback } \\
\text { (corrective, } \\
\text { praise) }\end{array}$ & $\begin{array}{l}\text { Outcome: Social interactions increased } \\
\text { modestly and high task engagement } \\
\text { maintained when job coaches reduced } \\
\text { proximity and delivered prompts } \\
\text { discretely. } \\
\text { Generalisation to daily life: yes } \\
\text { Follow-up: not measured }\end{array}$ & $4 / 5$ \\
\hline 10 & $\begin{array}{l}\text { Goodson } \\
\text { et al. (2007) } \\
{[51]}\end{array}$ & Setting a table & $\mathrm{n} / \mathrm{a}$ & $\mathrm{n} / \mathrm{a}$ & $\begin{array}{l}\text { Instruction } \\
\text { Modelling }\end{array}$ & $\begin{array}{l}\text { Feedback } \\
\text { (corrective) }\end{array}$ & $\begin{array}{l}\text { Outcome: Accuracy in setting the table } \\
\text { improved from } 0-60 \% \text { (baseline) to } 100 \% \\
\text { after a video prompting plus error } \\
\text { correction procedure. } \\
\text { Generalisation to daily life: yes } \\
\text { Follow-up: not measured }\end{array}$ & $2 / 5$ \\
\hline 11 & $\begin{array}{l}\text { Hällgren } \\
\text { et al. (2005) } \\
{[52]}\end{array}$ & $\begin{array}{l}\text { Activities of Daily } \\
\text { Living (ADL) }\end{array}$ & $\mathrm{n} / \mathrm{a}$ & $\mathrm{n} / \mathrm{a}$ & Unknown & Feedback $\left(\mathrm{NOS}^{\mathrm{a}}\right)$ & $\begin{array}{l}\text { Outcome: ADL performance improved in } \\
\text { five out of six participants after the } \\
\text { intervention, both regarding motor and } \\
\text { process skills. } \\
\text { Generalisation to daily life: yes } \\
\text { Follow-up: Improvements were } \\
\text { maintained. }\end{array}$ & $3 / 5$ \\
\hline
\end{tabular}


Table 2 (Continued)

\begin{tabular}{|c|c|c|c|c|c|c|c|c|}
\hline \multirow[t]{3}{*}{ \# } & \multirow{3}{*}{$\begin{array}{l}\text { Authors } \\
\text { (year) }\end{array}$} & \multirow[t]{3}{*}{ Target behaviour } & \multicolumn{4}{|c|}{ Behaviour Change Techniques } & \multirow[t]{3}{*}{ Main results } & \multirow{3}{*}{$\begin{array}{l}\text { Quality } \\
\text { appraisal }\end{array}$} \\
\hline & & & \multicolumn{2}{|c|}{ Applied by participant } & \multicolumn{2}{|c|}{ Applied by provider } & & \\
\hline & & & Antecedent & Consequent & Antecedent & Consequent & & \\
\hline 12 & $\begin{array}{l}\text { Johnson et al. } \\
\text { (1981) [53] }\end{array}$ & $\begin{array}{l}\text { Cooking skills: } \\
\text { broiling, baking, } \\
\text { boiling }\end{array}$ & $\mathrm{n} / \mathrm{a}$ & $\mathrm{n} / \mathrm{a}$ & $\begin{array}{l}\text { Instruction } \\
\text { Modelling } \\
\text { Physical } \\
\text { guidance } \\
\text { Prompts }\end{array}$ & $\begin{array}{l}\text { Feedback } \\
\text { (descriptive, } \\
\text { praise) } \\
\text { Reinforcement } \\
\text { (reward) }\end{array}$ & $\begin{array}{l}\text { Outcome: Relatively quick acquisition of } \\
\text { cooking skills once training initiated. } \\
\text { Three out of four participants showed } \\
\text { generalisation effects within and between } \\
\text { the cooking methods. } \\
\text { Generalisation to daily life: not measured } \\
\text { Follow-up: Relatively high maintenance of } \\
\text { cooking skills. }\end{array}$ & $2 / 5$ \\
\hline 13 & $\begin{array}{l}\text { Katz et al. } \\
\text { (1986) [54] }\end{array}$ & Fire-safety skills & $\mathrm{n} / \mathrm{a}$ & $\begin{array}{l}\text { Feedback } \\
\text { (praise) }\end{array}$ & $\begin{array}{l}\text { Instruction } \\
\text { Modelling } \\
\text { Physical } \\
\text { guidance } \\
\text { Prompts }\end{array}$ & $\begin{array}{l}\text { Feedback } \\
\text { (corrective, } \\
\text { descriptive, } \\
\text { praise) } \\
\text { Reinforcement } \\
\text { (reward) }\end{array}$ & $\begin{array}{l}\text { Outcome: For most participants, perfect } \\
\text { mastery of fire-safety skills after training } \\
\text { and significantly increased knowledge and } \\
\text { understanding of fire-safety behaviour. } \\
\text { Generalisation to daily life: yes } \\
\text { Follow-up: Most participants maintained } \\
\text { perfect mastery. }\end{array}$ & $2 / 5$ \\
\hline 14 & $\begin{array}{l}\text { Kottorp et al. } \\
(2003) \text { [55] }\end{array}$ & $\begin{array}{l}\text { Activities of Daily } \\
\text { Living (ADL) }\end{array}$ & $\mathrm{n} / \mathrm{a}$ & $\mathrm{n} / \mathrm{a}$ & $\begin{array}{l}\text { Compensatory } \\
\text { techniques } \\
\left(\mathrm{NOS}^{\mathrm{a}}\right) \\
\text { Environmental } \\
\text { restructuring }\end{array}$ & Feedback $\left(\mathrm{NOS}^{\mathrm{a}}\right)$ & $\begin{array}{l}\text { Outcome: The intervention had different } \\
\text { impacts across participants. Generally, } \\
\text { ADL process ability improved, but effects } \\
\text { on ADL motor ability and awareness of } \\
\text { disability were questionable. } \\
\text { Generalisation to daily life: yes } \\
\text { Follow-up: Improvements were maintained. }\end{array}$ & $1 / 5$ \\
\hline 15 & $\begin{array}{l}\text { LaCampagne } \\
\text { et al. (1987) } \\
{[56]}\end{array}$ & Paying bills & $\mathrm{n} / \mathrm{a}$ & $\mathrm{n} / \mathrm{a}$ & $\begin{array}{l}\text { Chaining } \\
\text { Instruction } \\
\text { Modelling }\end{array}$ & Feedback $\left(\mathrm{NOS}^{\mathrm{a}}\right)$ & $\begin{array}{l}\text { Outcome: Few errors in paying bills after } \\
\text { training, compared to many errors at } \\
\text { baseline. Skills generalised to untrained } \\
\text { bills. } \\
\text { Generalisation to daily life: not measured } \\
\text { Follow-up: Skills were maintained. }\end{array}$ & $2 / 5$ \\
\hline 16 & $\begin{array}{l}\text { Lovett et al. } \\
\text { (1989) [57] }\end{array}$ & $\begin{array}{l}\text { Activities of Daily } \\
\text { Living (ADL) }\end{array}$ & $\mathrm{n} / \mathrm{a}$ & $\begin{array}{l}\text { Feedback } \\
\left(\text { NOS }^{\text {a }}\right) \\
\text { Self- } \\
\text { evaluation } \\
\text { Self-recording } \\
\text { Self- } \\
\text { reinforcement }\end{array}$ & $\begin{array}{l}\text { Instruction } \\
\text { Modelling }\end{array}$ & $\begin{array}{l}\text { Feedback }\left(\mathrm{NOS}^{\mathrm{a}}\right) \\
\text { Reinforcement } \\
\text { (reward) }\end{array}$ & $\begin{array}{l}\text { Outcome: Improved ADL performance } \\
\text { compared to baseline. Group } 2 \text { (several BCTs) } \\
\text { generally performed better than Group } 1 \\
\text { (self-recording only) and performed slightly } \\
\text { better during maintenance phase, but } \\
\text { received more training. } \\
\text { Generalisation to daily life: yes } \\
\text { Follow-up: Higher ADL task completion for } \\
\text { all participants during long-term follow-up } \\
\text { vs. baseline. }\end{array}$ & $3 / 5$ \\
\hline 17 & $\begin{array}{l}\text { Marchetti } \\
\text { et al. (1983) } \\
\text { [58] }\end{array}$ & Pedestrian skills & $\mathrm{n} / \mathrm{a}$ & $\mathrm{n} / \mathrm{a}$ & $\begin{array}{l}\text { Generalisation } \\
\text { Prompts }\end{array}$ & $\begin{array}{l}\text { Feedback (praise) } \\
\text { Reinforcement } \\
\left(\text { social } \text { NOS }^{\mathrm{a}}\right)\end{array}$ & $\begin{array}{l}\text { Outcome: Community training group } \\
\text { significantly improved pedestrian skills. } \\
\text { No significant change in the Classroom } \\
\text { group. } \\
\text { Generalisation to daily life: yes } \\
\text { Follow-up: not measured }\end{array}$ & $1 / 5$ \\
\hline 18 & $\begin{array}{l}\text { Martin et al. } \\
\text { (1987) [59] }\end{array}$ & $\begin{array}{l}\text { Preparation of } \\
\text { breakfast and } \\
\text { dinner }\end{array}$ & Self-instruction & $\mathrm{n} / \mathrm{a}$ & $\begin{array}{l}\text { Instruction } \\
\text { Prompts }\end{array}$ & $\begin{array}{l}\text { Feedback } \\
\text { (corrective, } \\
\text { praise) }\end{array}$ & $\begin{array}{l}\text { Outcome: Rapid improvement in food } \\
\text { preparation skills with the use of picture } \\
\text { recipe cards. } \\
\text { Generalisation to daily life: yes } \\
\text { Follow-up: Two participants maintained } \\
\text { high performance, for the other it was } \\
\text { variable, but satisfactory. }\end{array}$ & $2 / 5$ \\
\hline 19 & $\begin{array}{l}\text { Matson } \\
\text { (1981) [60] }\end{array}$ & Shopping skills & $\mathrm{n} / \mathrm{a}$ & $\begin{array}{l}\text { Self- } \\
\text { evaluation }\end{array}$ & $\begin{array}{l}\text { Instruction } \\
\text { Modelling }\end{array}$ & $\begin{array}{l}\text { Feedback } \\
\text { (descriptive, } \\
\text { praise) }\end{array}$ & $\begin{array}{l}\text { Outcome: Intervention group improved } \\
\text { shopping skills, which generalised to } \\
\text { another store. The control group did not } \\
\text { improve. } \\
\text { Generalisation to daily life: yes } \\
\text { Follow-up: Intervention group } \\
\text { maintained gains and generalisation } \\
\text { effects. }\end{array}$ & $2 / 5$ \\
\hline 20 & $\begin{array}{l}\text { Matson } \\
(1982)[61]\end{array}$ & $\begin{array}{l}\text { Phone } \\
\text { conversational } \\
\text { skills }\end{array}$ & $\mathrm{n} / \mathrm{a}$ & $\begin{array}{l}\text { Self- } \\
\text { evaluation } \\
\text { Self- } \\
\text { monitoring }\end{array}$ & $\begin{array}{l}\text { Instruction } \\
\text { Modelling } \\
\text { Shaping }\end{array}$ & $\begin{array}{l}\text { Feedback } \\
\text { (corrective, } \\
\text { descriptive, } \\
\text { praise) } \\
\text { Reinforcement } \\
\left(\text { social NOS }{ }^{\mathrm{a}} \text { ) }\right.\end{array}$ & $\begin{array}{l}\text { Outcome: Independence training group } \\
\text { had better conversational skills than the } \\
\text { modelling and the control group. } \\
\text { Modelling group performed better than } \\
\text { the control group. } \\
\text { Generalisation to daily life: not measured. } \\
\text { Follow-up: Results were similar to } \\
\text { immediate outcomes. }\end{array}$ & $4 / 5$ \\
\hline 21 & $\begin{array}{l}\text { Matson et al. } \\
\text { (1986) [62] }\end{array}$ & $\begin{array}{l}\text { Computational } \\
\text { and shopping } \\
\text { skills }\end{array}$ & $\mathrm{n} / \mathrm{a}$ & $\begin{array}{l}\text { Self- } \\
\text { evaluation }\end{array}$ & $\begin{array}{l}\text { Instruction } \\
\text { Modelling }\end{array}$ & $\begin{array}{l}\text { Feedback } \\
\text { (descriptive, } \\
\text { praise) } \\
\text { Reinforcement } \\
\text { (social and } \\
\text { tangible NOS }{ }^{\mathrm{a}} \text { ) }\end{array}$ & $\begin{array}{l}\text { Outcome: Computational and shopping } \\
\text { skills rapidly improved after initiation of } \\
\text { intervention. Skills generalised to other } \\
\text { stores. } \\
\text { Generalisation to daily life: yes } \\
\text { Follow-up: Improvements were } \\
\text { maintained. }\end{array}$ & $2 / 5$ \\
\hline
\end{tabular}


Table 2 (Continued)

\begin{tabular}{|c|c|c|c|c|c|c|c|c|}
\hline \multirow[t]{3}{*}{ \# } & \multirow{3}{*}{$\begin{array}{l}\text { Authors } \\
\text { (year) }\end{array}$} & \multirow[t]{3}{*}{ Target behaviour } & \multicolumn{4}{|c|}{ Behaviour Change Techniques } & \multirow[t]{3}{*}{ Main results } & \multirow{3}{*}{$\begin{array}{l}\text { Quality } \\
\text { appraisal }\end{array}$} \\
\hline & & & \multicolumn{2}{|c|}{ Applied by participant } & \multicolumn{2}{|c|}{ Applied by provider } & & \\
\hline & & & Antecedent & Consequent & Antecedent & Consequent & & \\
\hline 22 & $\begin{array}{l}\text { Mclnerney } \\
\text { et al. (1992) } \\
{[63]}\end{array}$ & Use of the bus & $\begin{array}{l}\text { Use of cues } \\
\text { (environmental } \\
\text { landmarks }\end{array}$ & $\mathrm{n} / \mathrm{a}$ & $\begin{array}{l}\text { Chaining } \\
\text { Generalisation }\end{array}$ & $\begin{array}{l}\text { Feedback } \\
\text { (corrective, } \\
\text { praise) }\end{array}$ & $\begin{array}{l}\text { Outcome: Only follow-up measures were } \\
\text { used. } \\
\text { Generalisation to daily life: yes } \\
\text { Follow-up: Participants maintained their } \\
\text { mobility skills regarding making leisure } \\
\text { outings by bus. }\end{array}$ & $1 / 5$ \\
\hline 23 & $\begin{array}{l}\text { Michie et al. } \\
\text { (1998) [64] }\end{array}$ & $\begin{array}{l}\text { Community living } \\
\text { skills }\end{array}$ & Self-instruction & $\mathrm{n} / \mathrm{a}$ & $\begin{array}{l}\text { Chaining } \\
\text { Instruction } \\
\text { Modelling } \\
\text { Prompts } \\
\text { Roleplay } \\
\text { Shaping }\end{array}$ & $\begin{array}{l}\text { Reinforcement } \\
\left(\text { social NOS }^{\mathrm{a}}\right)\end{array}$ & $\begin{array}{l}\text { Outcome: In-vivo training group } \\
\text { performed better on community living } \\
\text { skills and adaptive behaviour vs. the other } \\
\text { groups, and scored higher on independent } \\
\text { functioning and socialisation vs. the } \\
\text { control group. Classroom group only } \\
\text { performed better regarding library use vs. } \\
\text { the control group. } \\
\text { Generalisation to daily life: yes } \\
\text { Follow-up: Results were similar to } \\
\text { immediate outcomes. }\end{array}$ & $2 / 5$ \\
\hline 24 & $\begin{array}{l}\text { Neef et al. } \\
\text { (1990) [65] }\end{array}$ & $\begin{array}{l}\text { Laundry skills } \\
\text { (washing and } \\
\text { drying) }\end{array}$ & $\mathrm{n} / \mathrm{a}$ & $\mathrm{n} / \mathrm{a}$ & $\begin{array}{l}\text { Generalisation } \\
\text { Modelling }\end{array}$ & $\begin{array}{l}\text { Feedback } \\
\text { (corrective, } \\
\text { praise) }\end{array}$ & $\begin{array}{l}\text { Outcome: Accuracy on the single case } \\
\text { machine improved from } 70-83 \% \text { at } \\
\text { baseline to } 100 \% \text { at post-training. } \\
\text { Performance on general case machines } \\
\text { improved from } 60-83 \% \text { to } 92-95 \% \text {. } \\
\text { Generalisation to untrained machines only } \\
\text { occurred after general case instruction. } \\
\text { Generalisation to daily life: yes } \\
\text { Follow-up: not measured }\end{array}$ & $2 / 5$ \\
\hline 25 & $\begin{array}{l}\text { Ores et al. } \\
(1984) \text { [66] }\end{array}$ & $\begin{array}{l}\text { Make a telephone } \\
\text { call }\end{array}$ & $\mathrm{n} / \mathrm{a}$ & $\mathrm{n} / \mathrm{a}$ & Modelling & $\mathrm{n} / \mathrm{a}$ & $\begin{array}{l}\text { Outcome: Nine out of ten participants } \\
\text { were able to dial successfully immediately } \\
\text { after training. } \\
\text { Generalisation to daily life: not measured } \\
\text { Follow-up: Results were similar to } \\
\text { immediate outcomes. }\end{array}$ & $2 / 5$ \\
\hline 26 & $\begin{array}{l}\text { Rehfeldt et al. } \\
\text { (2003) [67] }\end{array}$ & $\begin{array}{l}\text { Making a } \\
\text { sandwich }\end{array}$ & $\mathrm{n} / \mathrm{a}$ & $\mathrm{n} / \mathrm{a}$ & Modelling & Feedback (praise) & $\begin{array}{l}\text { Outcome: Rapid mastery of meal } \\
\text { preparation skill once training initiated. } \\
\text { Skill generalisation across settings. } \\
\text { Generalisation to daily life: not measured } \\
\text { Follow-up: Skill was maintained. }\end{array}$ & $2 / 5$ \\
\hline 27 & $\begin{array}{l}\text { Richman } \\
\text { et al. (1984) } \\
{[68]}\end{array}$ & $\begin{array}{l}\text { Menstrual care } \\
\text { skills }\end{array}$ & $\mathrm{n} / \mathrm{a}$ & $\mathrm{n} / \mathrm{a}$ & $\begin{array}{l}\text { Chaining } \\
\text { Instruction } \\
\text { Prompts }\end{array}$ & $\begin{array}{l}\text { Feedback } \\
\text { (corrective, } \\
\text { praise) }\end{array}$ & $\begin{array}{l}\text { Outcome: Performance improved } \\
\text { substantially after training initiated. } 100 \% \\
\text { correct performance maintained on the } \\
\text { validation and maintenance session. } \\
\text { Generalisation to daily life: yes } \\
\text { Follow-up: Maintenance of a high level of } \\
\text { responding. }\end{array}$ & $3 / 5$ \\
\hline 28 & $\begin{array}{l}\text { Risley et al. } \\
\text { (1980) [69] }\end{array}$ & $\begin{array}{l}\text { Making an } \\
\text { emergency call }\end{array}$ & $\mathrm{n} / \mathrm{a}$ & $\mathrm{n} / \mathrm{a}$ & $\begin{array}{l}\text { Generalisation } \\
\text { Instruction } \\
\text { Modelling } \\
\text { Physical } \\
\text { guidance }\end{array}$ & $\begin{array}{l}\text { Feedback } \\
\text { (corrective, } \\
\text { descriptive, } \\
\text { praise) }\end{array}$ & $\begin{array}{l}\text { Outcome: Performance improved after } \\
\text { training initiated. Skill generalisation to } \\
\text { other emergency parties. } \\
\text { Generalisation to daily life: not measured } \\
\text { Follow-up: Results were similar to } \\
\text { immediate outcomes }\end{array}$ & $2 / 5$ \\
\hline 29 & $\begin{array}{l}\text { Sarber et al. } \\
\text { (1983) [70] }\end{array}$ & $\begin{array}{l}\text { Menu planning } \\
\text { and grocery } \\
\text { shopping }\end{array}$ & $\mathrm{n} / \mathrm{a}$ & $\mathrm{n} / \mathrm{a}$ & $\begin{array}{l}\text { Instruction } \\
\text { Modelling } \\
\text { Prompt }\end{array}$ & $\begin{array}{l}\text { Feedback } \\
\text { (corrective, } \\
\text { praise) }\end{array}$ & $\begin{array}{l}\text { Outcome: Improved performance on } \\
\text { planning nutritious meals from } 0 \% \text { to } \\
100 \% \text { after completion of intervention. } \\
\text { Grocery shopping skills improved from } \\
25-72.5 \% \text { to } 100 \% \text { post-intervention. } \\
\text { Generalisation to daily life: yes } \\
\text { Follow-up: Meal planning and grocery } \\
\text { shopping skills varied from } 92.5-100 \% \text {. }\end{array}$ & $2 / 5$ \\
\hline 30 & $\begin{array}{l}\text { Sigafoos et al. } \\
(2005)[3]\end{array}$ & $\begin{array}{l}\text { Make a bag of } \\
\text { microwave } \\
\text { popcorn }\end{array}$ & $\mathrm{n} / \mathrm{a}$ & $\mathrm{n} / \mathrm{a}$ & $\begin{array}{l}\text { Instruction } \\
\text { Modelling }\end{array}$ & $\mathrm{n} / \mathrm{a}$ & $\begin{array}{l}\text { Outcome: Two out of three participants } \\
\text { improved from } 0-30 \% \text { at baseline to } 100 \% \\
\text { independence after video prompting started. } \\
\text { Generalisation to daily life: no } \\
\text { Follow-up: Independence ranged from } \\
80-100 \% \text {. }\end{array}$ & $1 / 5$ \\
\hline 31 & $\begin{array}{l}\text { Taber- } \\
\text { Doughty } \\
\text { et al. (2010) } \\
\text { [71] }\end{array}$ & Household tasks & $\mathrm{n} / \mathrm{a}$ & $\mathrm{n} / \mathrm{a}$ & $\begin{array}{l}\text { Instruction } \\
\text { Prompts }\end{array}$ & $\mathrm{n} / \mathrm{a}$ & $\begin{array}{l}\text { Outcome: Slightly more independent } \\
\text { performance when prompted by a telecare } \\
\text { provider vs. a standard care provider. } \\
\text { Generalisation to daily life: yes } \\
\text { Follow-up: Results were variable. }\end{array}$ & $2 / 5$ \\
\hline 32 & $\begin{array}{l}\text { Tam et al. } \\
(2005) \text { [72] }\end{array}$ & $\begin{array}{l}\text { Supermarket } \\
\text { shopping }\end{array}$ & $\mathrm{n} / \mathrm{a}$ & $\mathrm{n} / \mathrm{a}$ & $\begin{array}{l}\text { Instruction } \\
\text { Modelling } \\
\text { Roleplay }\end{array}$ & $\begin{array}{l}\text { Feedback }\left(\mathrm{NOS}^{\mathrm{a}}\right) \\
\text { Reinforcement } \\
\left(\text { verbal } \mathrm{NOS}^{\mathrm{a}}\right)\end{array}$ & $\begin{array}{l}\text { Outcome: Conventional training and } \\
\text { Virtual Reality (VR) group showed a } \\
\text { significant and similar improvement in } \\
\text { shopping skills. } \\
\text { Generalisation to daily life: yes } \\
\text { Follow-up: not measured }\end{array}$ & $2 / 5$ \\
\hline
\end{tabular}


Table 2 (Continued)

\begin{tabular}{|c|c|c|c|c|c|c|c|c|}
\hline \multirow[t]{3}{*}{$\#$} & \multirow{3}{*}{$\begin{array}{l}\text { Authors } \\
\text { (year) }\end{array}$} & \multirow[t]{3}{*}{ Target behaviour } & \multicolumn{4}{|c|}{ Behaviour Change Techniques } & \multirow[t]{3}{*}{ Main results } & \multirow{3}{*}{$\begin{array}{l}\text { Quality } \\
\text { appraisal }\end{array}$} \\
\hline & & & \multicolumn{2}{|c|}{ Applied by participant } & \multicolumn{2}{|c|}{ Applied by provider } & & \\
\hline & & & Antecedent & Consequent & Antecedent & Consequent & & \\
\hline 33 & $\begin{array}{l}\text { Taylor et al. } \\
\text { (1997) [73] }\end{array}$ & Shopping & Self-instruction & $\begin{array}{l}\text { Feedback } \\
\text { (descriptive, } \\
\text { praise) }\end{array}$ & $\begin{array}{l}\text { Instruction } \\
\text { Modelling } \\
\text { Prompts }\end{array}$ & $\mathrm{n} / \mathrm{a}$ & $\begin{array}{l}\text { Outcome: In Phase } 1 \text {, successful } \\
\text { performance of shopping task across } \\
\text { training and generalisation stores using } \\
\text { overt and covert self-instruction. Blocking } \\
\text { of overt and covert self-instruction } \\
\text { resulted in reversal to baseline levels. In } \\
\text { Phase } 2 \text {, successful performance of } \\
\text { shopping task using self-rules. High levels } \\
\text { of task analysis responding corresponded } \\
\text { with high levels of self-instruction. Similar } \\
\text { results in the generalisation settings. } \\
\text { Generalisation to daily life: yes } \\
\text { Follow-up: not measured }\end{array}$ & $2 / 5$ \\
\hline 34 & $\begin{array}{l}\text { Taylor et al. } \\
(2000) \text { [74] }\end{array}$ & $\begin{array}{l}\text { Supermarket } \\
\text { shopping }\end{array}$ & $\mathrm{n} / \mathrm{a}$ & $\mathrm{n} / \mathrm{a}$ & $\begin{array}{l}\text { Generalisation } \\
\text { Instruction } \\
\text { Modelling } \\
\text { Physical } \\
\text { guidance } \\
\text { Prompts } \\
\text { Roleplay }\end{array}$ & $\begin{array}{l}\text { Feedback } \\
\text { (corrective, praise }\end{array}$ & $\begin{array}{l}\text { Outcome: All participants reached } \\
\text { criterion responding in supermarket } \\
\text { training settings with little difference } \\
\text { between groups. Stimulus equivalence } \\
\text { training and Multiple exemplar training } \\
\text { were equally effective in promoting } \\
\text { generalisation, single instance training } \\
\text { was the least effective. } \\
\text { Generalisation to daily life: yes } \\
\text { Follow-up: not measured }\end{array}$ & $2 / 5$ \\
\hline 35 & $\begin{array}{l}\text { Wacker et al. } \\
\text { (1986) [75] }\end{array}$ & Clerical Tasks & $\mathrm{n} / \mathrm{a}$ & $\mathrm{n} / \mathrm{a}$ & Modelling & $\begin{array}{l}\text { Feedback } \\
\text { (corrective, } \\
\text { praise) }\end{array}$ & $\begin{array}{l}\text { Outcome: } 90 \text { \% of sheets were copied } \\
\text { correctly. Substantial increase in } \\
\text { incidental behaviours, indicating a more } \\
\text { appropriate interaction with the work } \\
\text { environment. } \\
\text { Generalisation to daily life: yes } \\
\text { Follow-up: not measured }\end{array}$ & $2 / 5$ \\
\hline 36 & $\begin{array}{l}\text { Wu et al. } \\
(2016) \text { [76] }\end{array}$ & Daily living skills & Self-instruction & $\mathrm{n} / \mathrm{a}$ & $\begin{array}{l}\text { Generalisation } \\
\text { Modelling } \\
\text { Physical } \\
\text { guidance } \\
\text { Prompts }\end{array}$ & $\begin{array}{l}\text { Feedback } \\
\text { (corrective) }\end{array}$ & $\begin{array}{l}\text { Outcome: All participants acquired a } \\
\text { variety of daily living skills using video } \\
\text { prompting. } \\
\text { Generalisation to daily life: yes } \\
\text { Follow-up: } 100 \% \text { accuracy was } \\
\text { maintained. }\end{array}$ & $4 / 5$ \\
\hline
\end{tabular}

$\mathrm{n} / \mathrm{a}=$ not applicable.

a Not Otherwise Specified.

with one group outperforming the other (article \#16, 20, 31). In two of these latter cases (article \#16, 20), the group with the most improvement received an intervention that used more BCTs than the other training group (e.g., not just self-recording, but also selfevaluation and self-reinforcement).

\section{Discussion and conclusion}

\subsection{Discussion}

This systematic review analysed studies on interventions that aim to promote self-management in daily life for adults with mild to moderate ID. We described the effectiveness of the interventions, as well as the BCTs that were used to attain the desired selfmanagement behaviour. In all the studies included, the authors reported that the self-management interventions were effective and that the positive effects were generally maintained over time. All studies that measured generalisation effects found that the selfmanagement behaviour trained was generalised to other tasks or (daily life) situations. With regard to the BCTs, all interventions involved BCTs applied by the provider of the intervention (e.g., trainer); in addition, one third of interventions also involved training participants with ID to apply BCTs themselves. Antecedent BCTs applied by participants included self-instructions and the use of cues; consequent BCTs included self-monitoring of the execution of the target behaviour and self-reinforcement. In most studies $(n=32)$, providers combined several antecedent BCTs, such as modelling, instructing, and prompting, with several consequent BCTs such as providing feedback or reinforcement. The findings seem to imply that interventions can promote self-management in people with mild to moderate ID, irrespective of the selfmanagement behaviour targeted and the characteristics of the intervention.

This review extends previous reviews that only analysed interventions for people with ID that targeted a specific selfmanagement domain [e.g., 18, 22] or BCT [e.g., 8, 24]. In line with previous reviews, the self-management interventions included showed positive results, but it was difficult to determine which factors contributed to the effectiveness of the interventions. Previous studies have suggested that a combination of multiple BCTs is most effective in promoting behaviour change $[18,31]$. However, it is as yet unknown which particular combinations might be especially effective for this population. Our finding that the interventions studied were considered effective is promising, as this suggests that people with mild to moderate ID can improve their self-management in daily life, regardless of the target behaviour, the specific intervention characteristics, and BCTs applied. It seems that as long as people with mild to moderate ID are provided with a self-management training, they are able to manage their affairs more independently, regardless of the type of affairs or self-management behaviour targeted. However, the finding that all interventions were reported to be effective also 
suggests a possible publication bias [32]. This type of bias is further increased because we only included articles and no book chapters or dissertations. In addition, studies were generally of moderate quality, which is common in the field of ID [27,33], and which further suggests a high risk of bias. Sample sizes were often very small, two thirds of studies used a (multiple) case study design; and only ten studies included a control group. Notably, quantitative data on results was often missing or incomplete. As a result, the interpretation and generalisation of the positive findings must be conducted with great caution, and hence no firm conclusions can be drawn.

Regarding the BCTs used to attain the targeted self-management behaviour, in the reviewed interventions it was found that in most cases BCTs were only applied by the intervention provider. This means that most of the time when people with ID were trained to promote their self-management, they were still largely dependent on the provider of the training. One could argue however, that a more effective and efficient way to promote overall self-management in people with ID is to teach them to apply BCTs or strategies themselves, such as self-instructions, self-rules, or general problem solving. This could reduce the need for proximity of a provider [34]. Especially since support staff already feel they cannot provide the quality of care that is needed for people with ID [35], a decreased dependence on the support provider is important to consider in self-management interventions. Furthermore, BCTs used by participants themselves can more easily be applied to other self-management tasks or situations [34,36-38], although whether this will occur may depend on the cognitive level of the person with ID.

If generalisation of BCTs is to be achieved, this needs to be targeted in interventions. However, even in the interventions reviewed in which BCTs were applied by participants, they only focused on the application of BCTs for specific behaviours. These behaviours often concerned very specific practical skills necessary at home or in the community, such as preparing food or withdrawing money. Looking at the quality of life domains as proposed by Schalock [39], the focus of self-management interventions for people with ID has mostly been limited to the domains of personal development, material wellbeing, and physical wellbeing. Domains such as interpersonal relations, self-determination, social inclusion, and rights, on the other hand, were hardly addressed in the interventions reviewed. Only three interventions targeted self-determination or rights [40-42], but these again only focused on a specific domain or context (e.g., making sexuality-related decisions). Therefore, to promote the overall quality of life of people with ID, interventions may need to go beyond training individual practical skills and may also need to focus on other important domains in life, such as self-management at work and in social interactions.

\subsection{Limitations}

One imitation of this study is that given the heterogeneity in study designs, types of interventions, and outcome measures, it was only possible to conduct a systematic review and not a metaanalysis,. In addition, we could not analyse which factors (e.g., participant or study characteristics) contributed to the effectiveness of interventions, and how and to what extent these factors contributed. We also could not analyse whether interventions in which BCTs were applied by participants with ID were more effective than those in which the BCTs were solely (or mainly) applied by the providers. Reasons for this were that all interventions were found to be effective, that sometimes only qualitative descriptions of results were reported, and that quantitative data (e.g., effect sizes) were often incomplete. Other important information was also often not reported. This included information regarding age, diagnosis of ID, recruitment of participants, inclusion and exclusion criteria, moments of assessment, any tailoring of interventions, the provider, the length and intensity of the intervention, and the BCTs applied. All this hampers the aggregation of data and thus the deduction of factors contributing to interventions' effectiveness, as well as a further examination of the specific groups of people with ID for which interventions are particularly effective. Also, the total sample size of all the studies reviewed was relatively small, which limits the generalisability of our findings. The abovementioned limitations commonly affect not only studies on self-management interventions, but also other types of studies in the field of ID, such as studies on lifestyle change interventions [27,33].

For future studies on self-management interventions we recommend providing more detailed information about the results and the participant and intervention characteristics. In addition, given the frequent occurrence in this field of relatively low-quality studies, there is a need for studies of high quality and with a low risk of bias (e.g., by including larger samples and applying randomisation techniques). Aspects to consider in future interventions could be the wider application of BCTs by people with ID themselves, with the aim of promoting overall self-management and quality of life, rather than solely targeting a particular practical skill. The transfer and generalisation of the target behaviour to daily life and across settings may also need to be incorporated in the interventions, as well as in the assessment of the intervention outcomes. Future studies could also further explore e-health or mhealth interventions [43], for example by creating self-management apps for tablets and mobile phones [44]. This may help people with ID to easily apply BCTs in all kinds of everyday situations without having to be dependent on the availability of their support staff and relatives [45]. For some people with ID this may require prior self-management training in using computers, tablets, and mobile phones, as well as in handling the internet and its potential dangers (e.g., unreliable websites and contacts) [44].

\subsection{Conclusion}

In sum, this review described a broad range of interventions for people with mild to moderate ID aimed at promoting their selfmanagement in daily life; at the same time, we evaluated the effectiveness of the interventions and the BCTs applied. Interventions generally targeted a particular skill by using a combination of several BCTs, mainly applied by the provider of the training. Although the results must be interpreted with caution due to the moderate methodological quality of most studies and the resulting high risk of bias, the finding that all interventions were reported to be effective seems to suggest that additional training can aid in the promotion of self-management in people with mild to moderate ID, regardless of the specific skill trained and the type of intervention provided. Further research is necessary to study the interventions' effectiveness more thoroughly, for example by examining what factors contribute to the effects of interventions and which type of intervention is effective for which subgroup of people with ID. This requires more specific information about the participants (e.g., diagnosis of ID, comorbidities) and the interventions (e.g., BCTs applied). Furthermore, it is recommended that self-management interventions target more diverse quality of life domains [39].

\section{Source of funding}

Funding for the study was provided by Raamwerk and Leiden University. This funding had no influence on the collection, analysis, and interpretation of the data; on the writing of the report; or on the decision to submit the article for publication. 


\section{Declaration of Competing Interest}

The authors declare that they have no conflict of interest.

\section{Acknowledgements}

We thank Jan Schoones of the Leiden University Medical Center for his help in developing the search strategy.

\section{Appendix A. Supplementary data}

Supplementary material related to this article can be found, in the online version, at doi:https://doi.org/10.1016/j.pec.2020.06.009.

\section{References}

[1] United Nations, Convention of the Rights of Persons With Persons With Disabilities, (2006)

[2] J. Sandjojo, W.A. Gebhardt, A.M.E.E. Zedlitz, J. Hoekman, J.A. den Haan, A.W.M Evers, Promoting independence of people with intellectual disabilities: A focus group study. Perspectives from people with intellectual disabilities, legal representatives, and support staff, J Policy Pract Intellect Disabil (in press).

[3] J. Sigafoos, M. O'Reilly, H. Cannella, M. Upadhyaya, C. Edrisinha, G.E. Lancioni, A Hundley, A. Andrews, C. Garver, D. Young, Computer-presented video prompting for teaching microwave oven use to three adults with developmental disabilities, J Behavl Educ 14 (2005) 189-201.

[4] T. Van Laarhoven, T. Van Laarhoven-Myers, Comparison of three video-based instructional procedures for teaching daily living skills to persons with developmental disabilities, Educ. Train. Dev. Disabil. 41 (2006) 365-381.

[5] American Psychiatric Association, Diagnostic and Statistical Manual of Menta Disorders, 5th ed., American Psychiatric Association, Washington, DC, 2013.

[6] J.C.E. Dusseljee, P.M. Rijken, M. Cardol, L.M.G. Curfs, P.P. Groenewegen, Participation in daytime activities among people with mild or moderate intellectual disability, J. Intellect. Disabil. Res. 55 (2011) 4-18.

[7] S. Ramdoss, R. Lang, C. Fragale, C. Britt, M. O’Reilly, J. Sigafoos, R. Didden, A. Palmen, G.E. Lancioni, Use of computer-based interventions to promote daily living skills in individuals with intellectual disabilities: a systematic review, J. Dev. Phys. Disabil. 24 (2012) 197-215.

[8] K.A. Smith, S.B. Shepley, J.L. Alexander, K.M. Ayres, The independent use of selfinstructions for the acquisition of untrained multi-step tasks for individuals with an intellectual disability: a review of the literature, Res. Dev. Disabil. 40 (2015) 19-30.

[9] R.J. Bond, J. Hurst, How adults with learning disabilities view living independently, Brit J Learn Disabil 38 (2010) 286-292.

[10] A. Haigh, D. Lee, C. Shaw, M. Hawthorne, S. Chamberlain, D.W. Newman, Z. Clarke, N. Beail, What things make people with a learning disability happy and satisfied with their lives: an inclusive research project, J. Appl. Res. Intellect. Disabil. 26 (2013) 26-33.

[11] N.M.J. Kuijken, J. Naaldenberg, M.W. Nijhuis-van der Sanden, H.M.J. Van Schrojenstein-Lantman de Valk, Healthy living according to adults with intellectual disabilities: towards tailoring health promotion initiatives, J. Intellect. Disabil. Res. 60 (2016) 228-241.

[12] C.A. Dollar, L.D. Fredrick, P.A. Alberto, J.K. Luke, Using simultaneous prompting to teach independent living and leisure skills to adults with severe intellectua disabilities, Res. Dev. Disabil. 33 (2012) 189-195.

[13] D. García-Villamisar, J. Dattilo, J.L. Matson, Quality of life as a mediator between behavioral challenges and autistic traits for adults with intellectual disabilities, Res. Autism Spectr. Disord. 7 (2013) 624-629.

[14] D.M. Browder, E.S. Shapiro, Applications of self-management to individuals with severe handicaps: a review, J. Assoc. Pers. Sev. Handicaps 10 (1985) 200 208.

[15] J. Sandjojo, A.M.E.E. Zedlitz, W.A. Gebhardt, J. Hoekman, E. Dusseldorp, J.A. den Haan, A.W.M. Evers, Training staff to promote self-management in people with intellectual disabilities, J. Appl. Res. Intellect. Disabil. 31 (2018) 840-850.

[16] M.L. Wehmeyer, K. Kelchner, S. Richards, Essential characteristics of self determined behavior of individuals with mental retardation, Am. J. Ment. Retard. 100 (1996) 632-642.

[17] M.L. Wehmeyer, B.H. Abery, Self-determination and choice, Intellect. Dev. Disabil. 51 (2013) 399-411.

[18] C.B. Gilson, E.W. Carter, E.E. Biggs, Systematic review of instructional methods to teach employment skills to secondary students with intellectual and developmental disabilities, Res. Pract. Persons Severe 42 (2017) 89107.

[19] G.E. Lancioni, M.F. O'Reilly, Self-management of instruction cues for occupation: review of studies with people with severe and profound developmental disabilities, Res. Dev. Disabil. 22 (2001) 41-65.

[20] F.R. Rusch, J. Dattilo, Employment and self-management: A. meta-evaluation of seven literature reviews, Intellect. Dev. Disabil. 50 (2012) 69-75.

[21] K. Storey, Review of research on self-management interventions in supported employment settings for employees with disabilities, Career Dev Except Individ 30 (2007) 27-34.
[22] M. Dannenberg, S.E. Mengoni, B. Gates, M. Durand, Self-management interventions for epilepsy in people with intellectual disabilities: a scoping review, Seizure 41 (2016) 16-25.

[23] L. Graham, J. Wright, R. Walwyn, A.M. Russell, L. Bryant, A. Farrin, A. House, Measurement of adherence in a randomised controlled trial of a complex intervention: supported self-management for adults with learning disability and type 2 diabetes, BMC Med. Res. Methodol. 16 (2016) 132.

[24] T. Koyama, H.T. Wang, Use of activity schedule to promote independent performance of individuals with autism and other intellectual disabilities: a review, Res. Dev. Disabil. 32 (2011) 2235-2242.

[25] S. Michie, S. Ashford, F.F. Sniehotta, S.U. Dombrowski, A. Bishop, D.P. French, A refined taxonomy of behaviour change techniques to help people change their physical activity and healthy eating behaviours: the CALO-RE taxonomy, Psychol. Health 26 (2011) 1479-1498.

[26] S. Michie, M. Richardson, M. Johnston, C. Abraham, J. Francis, W. Hardeman, M. P. Eccles, J. Cane, C.E. Wood, The behavior change technique taxonomy (v1) of 93 hierarchically clustered techniques: building an international consensus for the reporting of behavior change interventions, Ann. Behav. Med. 46 (2013) 81-95.

[27] M. Willems, T.I.M. Hilgenkamp, E. Havik, A. Waninge, C.A. Melville, Use of behaviour change techniques in lifestyle change interventions for people with intellectual disabilities: a systematic review, Res. Dev. Disabil. 60 (2017) 256268.

[28] D. Moher, A. Liberati, J. Tetzlaff, D.G. Altman, Prisma Group, Preferred reporting items for systematic reviews and meta-analyses: the PRISMA statement, PLoS Med. 6 (2009)e1000097.

[29] Q.N. Hong, P. Pluye, S. Fàbregues, G. Bartlett, F. Boardman, M. Cargo, P. Dagenais, M. Gagnon, F. Griffiths, B. Nicolau, A. O'Cathain, M. Rousseau, I. Vedel, Mixed Methods Appraisal Tool (MMAT) Version 2018 - User Guide, (2018) mixedmethodsappraisaltoolpublic.pbworks.com. (Accessed 01 Dec 2019..

[30] R. Didden, P. Duker, D. Seys, Gedragsanalyse En -therapie Bij Mensen Met Een Verstandelijke Beperking, 2nd ed., Elsevier gezondheidszorg, Maarssen, 2003.

[31] T.L. Webb, J. Joseph, L. Yardley, S. Michie, Using the internet to promote health behavior change: a systematic review and meta-analysis of the impact of theoretical basis, use of behavior change techniques, and mode of delivery on efficacy, J. Med. Internet Res. 12 (2010) 1-18.

[32] K. Dwan, D. Altman, J. Arnaiz, J. Bloom, A. Chan, E. Cronin, E. Decullier, P. Easterbrook, E. Von Elm, C. Gamble, D. Ghersi, J. Ioannidis, J. Simes, P. Williamson, Systematic review of the empirical evidence of study publication bias and outcome reporting bias, PLoS One 3 (2008) e3081.

[33] K.A. Smith, K.A. Ayres, J. Alexander, J.R. Ledford, C. Shepley, S.B. Shepley, Initiation and generalization of self-instructional skills in adolescents with autism and intellectual disability, J. Autism Dev. Disord. 46 (2016) 1196-1209.

[34] M.A. Hermsen, P. Embregts, A.H.C. Hendriks, N. Frielink, The human degree of care. Professional loving care for people with a mild intellectual disability: an explorative study, J. Intellect. Disabil. Res. 58 (2014) 221-232.

[35] A.E. Harchik, J.A. Sherman, J.B. Sheldon, The use of self-managment procedures by people with developmental disabilities: a brief review, Res. Dev. Disabil. 13 (1992) 211-227.

[36] B.J. Faloon, R.A. Rehfeldt, The role of overt and covert self-rules in establishing a daily living skill in adults with mild developmental disabilities, J. Appl. Behav. Anal. 41 (2008) 393-404.

[37] M.A. Mastropieri, T.E. Scruggs, R.L. Shiah, Can computers teach problemsolving strategies to students with mild mental retardation? A case study, Remedial Spec. Educ. 18 (1997) 157-165.

[38] R.L. Schalock, The concept of quality of life: what we know and do not know, J Intellect. Disabil. Res. 48 (2004) 203-216.

[39] E. Dukes, B.E. McGuire, Enhancing capacity to make sexuality-related decisions in people with an intellectual disability, J. Intellect. Disabil. Res. 53 (2009) 727-734.

[40] G.D. Faw, P.K. Davis, C. Peck, Increasing self-determination: teaching people with mental retardation to evaluate residential options, J. Appl. Behav. Anal. 29 (1996) 173-188.

[41] M.A. Feldman, F. Owen, A. Andrews, J. Hamelin, R. Barber, D. Griffiths, Health self-advocacy training for persons with intellectual disabilities, J. Intellect. Disabil. Res. 56 (2012) 1110-1121.

[42] M. Willems, A. Waninge, T.I.M. Hilgenkamp, P. van Empelen, W.P. Krijnen, C.P. van der Schans, C.A. Melville, Effects of lifestyle change interventions for people with intellectual disabilities: systematic review and meta-analysis of randomized controlled trials, J. Appl. Res. Intellect. Disabil. 31 (2018) 949-961.

[43] A. Vázquez, C. Jenaro, N. Flores, M.J. Bagnato, M.C. Pérez, M. Cruz, E-health interventions for adult and aging population with intellectual disability: a review, Front. Psychol. 9 (2018).

[44] N. Frielink, C.E.M. Oudshoorn, P.J.C.M. Embregts, e-health in support for daily functioning of people with intellectual disability: views of service users, relatives, and professionals on both its advantages, disadvantages and its facilitating and impeding factors, Intellect. Dev. Disabil. (2020).

[45] B. Wennberg, A. Kjellberg, Participation when using cognitive assistive devices - from the perspective of people with intellectual disabilities, Occup. Ther. Int. 17 (2010) 168-176.

[46] K.A. Crnic, H.A. Pym, Training mentally retarded adults in independent living skills, Ment. Retard. 17 (1979) 13-16.

[47] D.K. Davies, S.E. Stock, M.L. Wehmeyer, Application of computer simulation to teach ATM access to individuals with intellectual disabilities, Educ. Train. Dev. Disabil. 38 (2003) 451-456. 
[48] D.K. Davies, S.E. Stock, S. Holloway, M.L. Wehmeyer, Evaluating a GPS-based transportation device to support independent bus travel by people with intellectual disability, Intellect. Dev. Disabil. 48 (2010) 454 463.

[49] M.A. Feldman, J.M. Ducharme, L. Case, Using self-instructional pictorial manuals to teach child-care skills to mothers with intellectual disabilities, Behav. Modif. 23 (1999) 480-497.

[50] C.B. Gilson, E.W. Carter, Promoting social interactions and job independence for college students with autism or intellectual disability: a pilot study, J. Autism Dev. Disord. 46 (2016) 3583-3596.

[51] J. Goodson, J. Sigafoos, M. O'Reilly, H. Cannella, G.E. Lancioni, Evaluation of a video-based error correction procedure for teaching a domestic skill to individuals with developmental disabilities, Res. Dev. Disabil. 28 (2007) 458467.

[52] M. Hällgren, A. Kottorp, Effects of occupational therapy intervention on activities of daily living and awareness of disability in persons with intellectual disabilities, Aust. Occup. Ther. J. 52 (2005) 350-359.

[53] B.F. Johnson, A.J. Cuvo, Teaching mentally retarded adults to cook, Behav. Modif. 5 (1981) 187-202.

[54] R.C. Katz, N.N. Singh, Comprehensive fire-safety training for adult mentally retarded persons, J. Ment. Defic. Res. 30 (1986) 59-69.

[55] A. Kottorp, M. Hallgren, B. Bernspang, A.G. Fisher, Client-centred occupational therapy for persons with mental retardation: implementation of an intervention programme in activities of daily living tasks, Scand. J. Occup. Ther 10 (2003) 51-60.

[56] J. LaCampagne, E. Cipani, Training adults with mental retardation to pay bills, Ment. Retard. 25 (1987) 293-303.

[57] D.L. Lovett, K.A. Haring, The effects of self-management training on the daily living of adults with mental retardation, Educ. Train. Dev. Disabil. 24 (1989) 306-323.

[58] A.G. Marchetti, J.R. McCartney, S. Drain, M. Hooper, J. Dix, Pedestrian skills training for mentally retarded adults: comparison of training in two settings, Ment. Retard. 21 (1983) 107-110.

[59] J.E. Martin, F.R. Rusch, Use of the partial-sequential withdrawal design to assess maintenance of mentally retarded adults' acquired meal preparation skills, Res. Dev. Disabil. 8 (1987) 389-399.

[60] J.L. Matson, Use of independence training to teach shopping skills to mildly mentally retarded adults, Am. J. Ment. Defic. 86 (1981) 178-183.

[61] J.L. Matson, Independence training vs modeling procedures for teaching phone conversation skills to the mentally retarded, Behav. Res. Ther. 20 (1982) 505-511.
[62] J.L. Matson, S. Long, Teaching computation/shopping skills to mentally retarded adults, Am. J. Ment. Defic. 91 (1986) 98-101.

[63] C.A. McInerney, M. McInerney, A mobility skills training program for adults with developmental disabilities, Am. J. Occup. Ther. 46 (1992) 233-239.

[64] A.M. Michie, W.R. Lindsay, A.H. Smith, J. Todman, Changes following community living skills training: a controlled study, Br. J. Clin. Psychol. 37 (Pt 1 ) (1998) 109-111.

[65] N.A. Neef, J. Lensbower, I. Hockersmith, V. DePalma, K. Gray, In vivo versus simulation training: an interactional analysis of range and type of training exemplars, J. Appl. Behav. Anal. 23 (1990) 447-458.

[66] L. Ores, L.H. Parent, Telephone Call Aid for problems of visual sequencing, Am. J. Occup. Ther. 38 (1984) 40-43.

[67] R.A. Rehfeldt, D. Dahman, A. Young, H. Cherry, P. Davis, Teaching a simple meal preparation skill to adults with moderate and severe mental retardation using video modeling, Behav. Interv. 18 (2003) 209-218.

[68] G.S. Richman, M.L. Reiss, K.E. Bauman, J.S. Bailey, Teaching menstrual care to mentally retarded women: acquisition, generalization, and maintenance, J. Appl. Behav. Anal. 17 (1984) 441-451.

[69] R. Risley, A.J. Cuvo, Training mentally retarded adults to make emergency telephone calls, Behav. Modif. 4 (1980) 513-525.

[70] R.E. Sarber, M.M. Halasz, M.C. Messmer, A.D. Bickett, J.R. Lutzker, Teaching menu planning and grocery shopping skills to a mentally retarded mother, Ment. Retard. 21 (1983) 101-106.

[71] T. Taber-Doughty, J. Shurr, J. Brewer, S. Kubik, Standard care and telecare services: comparing the effectiveness of two service systems with consumers with intellectual disabilities, J. Intellect. Disabil. Res. 54 (2010) 843-859.

[72] S.F. Tam, D.W.-K. Man, Y.P. Chan, P.C. Sze, C.M. Wong, Evaluation of a computerassisted, 2-D virtual reality system for training people with intellectual disabilities on how to shop, Rehabil. Psychol. 50 (2005) 285-291.

[73] I. Taylor, M.F. O'Reilly, Toward a functional analysis of private verbal selfregulation, J. Appl. Behav. Anal. 30 (1997) 43-58.

[74] I. Taylor, M.F. O'Reilly, Generalization of supermarket shopping skills for individuals with mild intellectual disabilities using stimulus equivalence training, Psychol. Rec. 50 (2000) 49-62.

[75] D.P. Wacker, W.K. Berg, M.B. Visser, J.E. Egan, A preliminary evaluation of independence in a competitive employment setting, J. Assoc. Pers. Sev. Handicaps 11 (1986) 246-254.

[76] P.F. Wu, J.E. Wheaton, H.I. Cannella-Malone, Effects of video prompting and activity schedules on the acquisition of independent living skills of students who are deaf and have developmental disabilities, Educ. Train. Autism Dev. Disabil. 51 (2016) 366-378. 